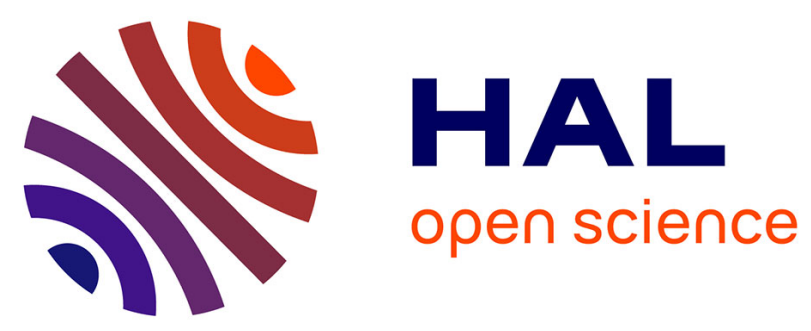

\title{
Pickering emulsions stabilized by thermoresponsive oligo(ethylene glycol)-based microgels: effect of temperature-sensitivity on emulsion stability
}

Marie-Charlotte Tatry, Paul Galanopoulo, Léa Waldmann, Véronique

Lapeyre, Patrick Garrigue, Véronique Schmitt, Valérie Ravaine

\section{To cite this version:}

Marie-Charlotte Tatry, Paul Galanopoulo, Léa Waldmann, Véronique Lapeyre, Patrick Garrigue, et al.. Pickering emulsions stabilized by thermoresponsive oligo(ethylene glycol)-based microgels: effect of temperature-sensitivity on emulsion stability. Journal of Colloid and Interface Science, In press, 589, pp.96-109. hal-03110385

\section{HAL Id: hal-03110385 \\ https://hal.science/hal-03110385}

Submitted on 14 Jan 2021

HAL is a multi-disciplinary open access archive for the deposit and dissemination of scientific research documents, whether they are published or not. The documents may come from teaching and research institutions in France or abroad, or from public or private research centers.
L'archive ouverte pluridisciplinaire HAL, est destinée au dépôt et à la diffusion de documents scientifiques de niveau recherche, publiés ou non, émanant des établissements d'enseignement et de recherche français ou étrangers, des laboratoires publics ou privés. 


\section{Pickering emulsions stabilized by}

\section{thermoresponsive oligo(ethylene glycol)-based}

\section{microgels: effect of temperature-sensitivity on}

\section{emulsion stability}

Marie-Charlotte Tatry ${ }^{1,2}$, Paul Galanopoulo ${ }^{1}$, Léa Waldmann ${ }^{1}$, Véronique Lapeyre ${ }^{1}$, Patrick Garrigue, , Véronique Schmitt*2, Valérie Ravaine*1

${ }^{1}$ Univ. Bordeaux, CNRS, Bordeaux INP, ISM, UMR 5255, F-33400, Talence, France

${ }^{2}$ Centre de Recherche Paul Pascal, UMR 5031, Université de Bordeaux, CNRS, 115 Avenue du Dr A. Schweitzer, 33600 Pessac, France.

* corresponding authors

Email: vravaine@enscbp.fr; Tel: +33-556846613

Email: veronique.schmitt@,crpp.cnrs.fr; Tel: +33-556845667 


\begin{abstract}
Hypothesis

The stability of emulsions stabilized by soft and responsive microgels and their macroscopic properties are governed by the microstructure of microgels, in particular their deformability. However, little is known about the role of the microgel chemistry, though it is expected that polymeric backbone with an amphiphilic structure is a requirement for their adsorption at the oil-water interface.
\end{abstract}

\title{
Experiments
}

A series of biocompatible, thermoresponsive and amphiphilic poly(oligoethylene glycol)methacrylate (pOEMA) microgels is synthesized, with varying hydrophobic-hydrophilic balance, or equivalent varying volume phase transition temperature (VPTT). Their behavior in the bulk phase and at solid interfaces is compared to their behavior at liquid interfaces, studied on flat and model interfaces by the pendant drop method, and on drops, in microgel-stabilized emulsions.

\section{Findings}

Controlling the composition of microgels by simply changing the number of ethylene oxide groups in the hydrophilic side chain allows a precise tuning of their VPTT in the range of 20 to $60^{\circ} \mathrm{C}$. Simultaneously, the swelling ratio and the deformability of the microgels increase by increasing the hydrophilicity, as a result of the polymerization process. Regardless of their hydrophilicity, all the swollen pOEMA microgels adsorb at the liquid interface and stabilize emulsions, whose flocculation state and mechanical stability depends on the microgel deformability. Unexpectedly, most emulsions remain stable upon heating above the VPTT of the microgels. Such feature highlights their extreme robustness, whose origin is discussed. This study opens new opportunities for the use of biocompatible Pickering emulsifiers. 
Keywords: Microgels, Pickering emulsions, Interfaces, thermoresponsive polymers, Biocompatible polymers. 


\section{Introduction}

Micro-and nanogels are particularly attractive particles for a broad range of applications in solution, but their ability to adsorb at interfaces is also highly promising [1]. This owes to the particle deformability which brings original properties at the interface compared to solid particles $[2,3,4]$. In particular, the adsorption of microgels at liquid interfaces has led to the stabilization of so-called Pickering emulsions and foams, which can be further destabilized on demand by triggering the volume phase transition of microgels from swollen to collapsed state [5-13]. To date, a great knowledge has been collected using poly( $N$-isopropylacrylamide $)$ as model microgels $[3,4,14]$. These colloidal cross-linked polymeric networks are made of amphiphilic chains, intrinsically related to their thermoresponsive behavior. Indeed, pNIPAM has a Lower Critical Solution Temperature (LCST) of $32^{\circ} \mathrm{C}$. The corresponding particles are swollen by water at low temperature, when hydrogen bondings between water and amide dominate, and collapse above the LCST, called the volume phase transition temperature (VPTT), when hydrogen bondings break down and hydrophobic interactions dominate [15]. Due to the nature of the backbone, such microgels adsorb at liquid interfaces and undergo chain expansion to minimize interfacial energy costs [16], being able to reach cross-sections higher than their initial dimensions in solution $[2,17]$. For single microgels, the degree of flattening depends on the deformability of microgels, the less cross-linked microgels being the more flexible. The microstructure of the microgels plays also an important role [18]. In the case of pNIPAM microgels prepared by batch process, the uneven distribution of the cross-linker gives rise to fried-egg structures, where the shell is more deformed than the core [2,19]. The presence of a thin corona of dangling chains at the periphery of microgels was confirmed both experimentally, by small angle neutron reflectivity [20] and theoretically [21]. The total volume of the microgel remains unchanged upon deformation $[22,23]$, meaning that the microgels are swollen by water and protrude in this medium, while they are unswollen by oil and lie flat on 
the oil side $[19,24]$. At the level of a microgel collection, conformational changes depend also on the interfacial concentration of microgels, a parameter which can easily be adjusted by interfacial compression, using a Langmuir trough technique [23,25-30]. Alternatively, in the case of spontaneous adsorption, the microgel density at the interface, at a given time, may be varied by changing the concentration in the bulk phase [31-34]. The presence of dangling chains is thought to have a major impact on lateral interactions between microgels, thereby creating entanglements between adjacent microgels. This should increase interfacial elasticity and could explain the origin of emulsion mechanical stability. Indeed, it was observed that increasing the cross-linking density of the microgels decreased emulsion mechanical stability [2]. This was attributed to a loss of connectivity between microgels owing to less dangling chains. Hence, the deformability and the microstructure have a great impact on emulsion stability.

Apart from these model pNIPAM microgels, efforts were carried out to extend the field of applications of such structures. The role of comonomers was explored in pNIPAM chemistry, enlarging the panel of stimuli: various N-alkylacrylamide derivatives to change the VPTT [9], carboxylic acid derivatives to induce pH-responsiveness [10], phenylboronates to introduce glucose-responsiveness [35]. Such studies lead to the conclusion that the main parameter to trigger emulsion stability was the deformability of microgels, which could be tuned by additional physical cross-links brought by the functional groups, whereas electrostatic interactions did not change the stability [36]. Among synthetic polymers, poly(Nvinylcaprolactam) (pVCL) microgels were successfully used as biocompatible and thermoresponsive substitute of pNIPAM [37]. Conversely, many microgels arise from biopolymers, making food science the most important field of application of the above concepts $[38,39]$. Yet, protein [40-44] or polysaccharide [45] microgels have been successfully used as emulsion or foam stabilizers. 
To date, a better understanding of the role of microgel chemical composition is still missing. A crucial question is the importance of polymer amphiphilicity. Since most of the fundamental studies were focused on pNIPAM chemistry, little is known about the role of the backbone chemical composition. How amphiphilic should the microgel structure be to allow efficient adsorption at the liquid interface and to provide emulsion stability? Moreover, the thermoresponsiveness of the microgels is intrinsically related to their hydrophilic-hydrophobic balance. The occurrence of the VPTT is a direct consequence of the domination of hydrophobic interactions over the formation of hydrogen bonds with hydrophilic groups. The VPTT increases when their hydrophilic character increases. Should it be expected that microgels having a higher VPTT adsorb less efficiently than those having a lower VPTT due to their lower affinity for the interface? To test these hypotheses and enlarge the field of applications of microgel-stabilized emulsions, we explore the chemistry of oligo(ethyleneglycol) (meth)acrylates, which has been widely used as an alternative to pNIPAM chemistry since the pioneering work of Lutz [46-48]. The range of available monomers, with various oligo(ethylene glycol) side chains, allows tuning the LCST of the polymer while respecting biocompatibility criterion $[49,50]$. Of particular interest for this study, is their chemical structure, represented in Fig. 1. It shows their intrinsic amphiphilicity, where the hydrophobic part refers to the methacrylate group and the hydrophilic part refers to the oligo(ethylene glycol) chain. The length of this latter block defines the affinity for water, and thus the value of the LCST. More precisely, the longer the ethylene glycol chain length, the higher the LCST. Various microgels based on this type of chemistry have already been developed [51-56] and used as drug delivery systems, either in suspensions [57] or in the form of skin-adhesive film [58,59]. Playing in the backyard of monomer selection, various systems were developed with modulated responsiveness, in particular exhibiting linear thermoresponsive behavior using acrylate groups $[53]$. 
In the present paper, we exploit this knowledge to prepare a series of pOEMA microgels with varying the VPTT and explore the link between their chemical composition/structure and their ability to stabilize oil-in-water emulsions. In particular, the deformability of microgels is questioned. The study is completed by the exploitation of microgel thermosensitivity as a trigger to tune the emulsion properties. Some similarities with model pNIPAM microgels are found but the complexity of the destabilization mechanisms is also demonstrated.

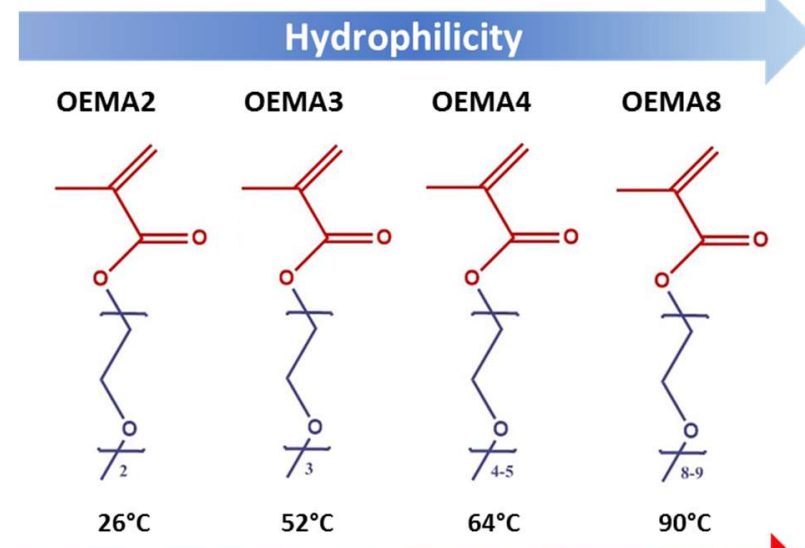

LCST of the corresponding polymer

Figure 1. Chemical structure of oligo ethylene glycol methacrylates monomers and the LCST values of the corresponding homopolymers (adapted from $[48,50]$ ).

\section{Material and methods}

\subsection{Chemicals}

All reagents were purchased from Sigma-Aldrich unless otherwise noted. Oligo(ethylene glycol) methyl ether methacrylate with various chain lengths and thus molecular weights were purchased and named OEMAn followed by the average number n of ethylene oxide (EO) units: di(ethylene glycol) methyl ether methacrylate (OEMA2, purity $95 \%, M_{n}=188 \mathrm{~g} / \mathrm{mol}$ ), tri(ethylene glycol) methyl ether methacrylate (OEMA3, purity $93 \%, M_{n}=232 \mathrm{~g} / \mathrm{mol}$ ), oligo(ethylene glycol) methyl ether methacrylate (OEMA4, 
having 4-5 EO units, $\mathrm{Mn}=300 \mathrm{~g} / \mathrm{mol}$ ), oligo(ethylene glycol) methyl ether methacrylate (OEMA8, having 8-9 EO units $\mathrm{M}_{\mathrm{n}}=500 \mathrm{~g} / \mathrm{mol}$ ). Oligo(ethylene glycol) diacrylate (OEDA, $\mathrm{Mn}=250 \mathrm{~g} / \mathrm{mol}$ ) was used as a cross-linker. The initiator, potassium persulfate (KPS) and the model oil n-dodecane (purity $>99 \%$ ) were used as received. Milli-Q water was used for all synthesis reactions, purification, and solution preparation.

\subsection{Particles synthesis and purification}

The synthesis procedure is based on radical precipitation polymerization similar to that of pNIPAM [60], adapted from previous work by Boularas et al. [61]. Polymerization was performed in a $100 \mathrm{~mL}$ three-neck round-bottom flask, equipped with a magnetic stir bar, a reflux condenser, thermometer and nitrogen inlet. The initial total monomer concentration was held constant at $95 \mathrm{mM}$, with a cross-linker concentration equal to $2 \mathrm{~mol} \%$. The rest of the monomers could be either a single monomer (OEMA2, 3 or 4), or a mixture of OEMA2 and OEMA8. The whole solution was heated at $90^{\circ} \mathrm{C}$ and thoroughly purged with nitrogen for 30 min prior to initiation. Free radical polymerization was then initiated with $2.5 \mathrm{mM}$ of KPS $(0.5$ $\mathrm{mM}$ in the case of single monomers) dissolved in $2 \mathrm{~mL}$ of water and degassed for $5 \mathrm{~min}$. The successful initiation was indicated by the occurrence of turbidity. The solution was allowed to react for a period of $4 \mathrm{~h}$ under nitrogen atmosphere. Following the synthesis, the microgels were purified by repeated centrifugation-redispersion steps in water (16 $000 \mathrm{rpm}$ corresponding to $29220 \mathrm{gr}_{\mathrm{r}}$ where $\mathrm{g}_{\mathrm{r}}$ is the terrestrial acceleration, for 1 hour, at $24^{\circ} \mathrm{C}$ ) until the surface tension of supernatant was above $70 \mathrm{mN} / \mathrm{m}$ for $1 \mathrm{~min}$ showing that the microgel dispersions were almost free of surface active impurities (approx. 6 cycles). The resulting microgels were named pOEMA $\mathrm{n}$ or pOEMA $2 / 8 \mathrm{x} \%$, where $\mathrm{x}$ is the molar percentage of OEMA 8 in the (OEMA 2+OEMA 8) synthesis mixture. The two routes are summarized in Fig. 2.

The particle mass $\mathrm{m}_{\text {part }}($ in $\mathrm{wt} \%$ ) in aqueous dispersions was determined by the drying method at $50^{\circ} \mathrm{C}$. 


\subsection{Particle characterization}

Dynamic Light Scattering. Hydrodynamic diameters and polydispersity index (PDI) were determined by dynamic light scattering (DLS) with a Zetasizer Nano S90 (Malvern Instruments). The samples contained about $0.01 \mathrm{wt} \%$ of polymer in aqueous solutions.

Colloidal stability. The colloidal stability of the microgel suspension was assessed by measuring the evolution of the hydrodynamic diameter as a function of temperature, in $\mathrm{NaCl}$ $0.1 \mathrm{M}$ solution. When microgels reached their collapsed state, they became flocculated leading to the divergence of the hydrodynamic diameter. The temperature corresponding to this divergence is called the flocculation temperature.

Transmission Electron Microscopy (TEM). The microgel morphology was studied by TEM using a TEM JEOL JEM $1400(120 \mathrm{kV})$, after depositing a drop of the dilute suspension on a copper grid coated with a carbon membrane, followed by staining with uranyl acetate.

\section{Atomic Force Microscopy (AFM)}

The dried microgels were observed by AFM (Veeco Multimode 8) after deposition on a silicon substrate-by drop casting. Topographic images were obtained in Scanasyst-in Air mode using a silicon tip on nitride lever with a spring constant of $0.4 \mathrm{~N} \cdot \mathrm{m}^{-1}$ and a resonance frequency 70 $\mathrm{kHz}$. Profile views of at least 10 microgels were analyzed.

\subsection{Emulsion preparation and characterization}

Emulsification. Emulsion batches composed of dodecane (30 wt\%) in an aqueous solution of microgels were prepared by emulsification at $4^{\circ} \mathrm{C}$ using an Ultra-Turrax T25 mixer, at constant speed of $9500 \mathrm{rpm}$ for $30 \mathrm{~s}$. The temperature during the emulsification step was chosen as low as possible in order to preclude microgel deswelling that could occur during shearing due to local heat generation.. Emulsion characterization was then performed at $25^{\circ} \mathrm{C}$. The macroscopic 
state of the emulsion was checked visually. Low microgel concentrations Cp-were chosen in order to explore the limited coalescence domain. This particle-poor regime leads to more monodisperse emulsions with total microgel adsorption at the oil/water interface [62]. Cp were in the range between 0.01 and $0.05 \mathrm{wt} \%$ with respect to water weight.

Optical microscopy. The size distribution of emulsions was estimated by direct imaging using inverted optical microscope (Zeiss Axiovert X100) and video camera.

CryoSEM observations. Cryo-SEM observations were carried out following the previously described procedure [35].

\subsection{Dynamic surface tension}

Dynamic interfacial tension of the air/water interface was measured using the pendant-drop technique (Teclis). An aqueous drop of $10 \mu \mathrm{L}\left(0.05 \mathrm{wt} \%\right.$ of microgels in $\left.\mathrm{NaCl} 10^{-2} \mathrm{M}\right)$ was suspended in air for over 10000 seconds, in a closed container filled with some water at the bottom in order to limit drop evaporation to less than $5 \%$ of the drop volume. The drop surface area was approximately $22 \mathrm{~mm}^{2}$. Salt was added to the solution in order to minimize any electrostatic contribution [34]. The tension was deduced from the axisymmetric drop shape by fitting it with the Laplace equation. .

\section{Results}

\subsection{Synthesis of microgels}

A series of pOEMA microgels with varying composition was prepared by free radical precipitation polymerization of OEMA monomers and OEDA. This process is very easy to handle experimentally and can yield batches of highly monodisperse microgels. However, the resulting microstructure of the particles is the consequence of various parameters, in particular the reactivity ratio between the comonomers but also the initiator concentration [63]. Moreover, 
all the monomers have to be soluble in water but the growing oligomers should precipitate at the polymerization temperature chosen above their LCST. The targeted LCST should therefore be lower than the polymerization temperature. In order to reach the largest panel of LCSTs, a unique polymerization temperature was chosen as high as possible i.e. $90^{\circ} \mathrm{C}$.

Two strategies were employed to tune the VPTT of the microgels (Fig. 2): either a mixture of two monomers, bearing different EO units, 2 and 8 (route 1) or single monomers bearing different number of EO units (2, 3 or 4) (route 2). Route 1 is generally employed in the literature because it gives a greater flexibility to finely adjust the VPTT of the microgels, by choosing the composition of the two comonomer mixture. But it gives additional complexity to the structure of the particles. It was shown that microgels made of copolymers bearing two different LCST presented nanophase separation between domains made of collapsed segments and domains made of swollen ones, for temperatures comprised between the two LCST [54, 64]. This also means that some parts of the polymers could be incompletely collapsed even above their VPTT. In order to avoid such complexity, route 2 leading to homopolymer microgels was also employed, although restricted to a few commercially-available monomers.

1)<smiles>C=C(C)C(=O)OCCOCCOC</smiles><smiles>C=C(C)C(=O)OC(C)(C)COC(C)(C)C</smiles>

2)<smiles>C=C(C)C(=O)OC(C)(C)COC(C)(C)C</smiles>

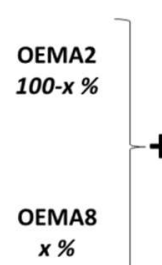

$n=2-3$ or 4<smiles>C=CC(=O)COCC(C)(C)OC(=O)C=CC(=O)OCC</smiles><smiles>C=CC(=O)OCC(C)(C)OC(=O)C=C</smiles>

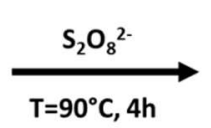
$\mathrm{T}=90^{\circ} \mathrm{C}, 4 \mathrm{~h}$ POEMA $2 / 8 \mathrm{x} \%$
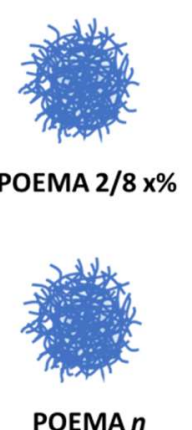

Figure 2. Synthesis of microgels showing the two strategies: 1) using a mixture of comonomers to tune the VPTT, 2) using a single monomer with varying OE chain length. 
OEDA was chosen as a cross-linker based on Boularas's previous work [61] ethylene glycol methacrylate (EGDMA) is more generally employed in other pOEMA microgels [51, 54]. Despite their different polymerization rates, SANS studies revealed that both cross-linkers lead to core-shell structures, the core being more cross-linked than the shell $[54,57]$. It was also observed that OEDA generates more water-soluble species [57].

Using route 1, all the microgels were successfully synthesized, up to a content of OEMA8 of $25 \%$. Above this content, the growing polymers were probably highly soluble in water and precipitation process did not occur. In the case of route 2, polymerization of OEMA4 required to reduce the amount of initiator otherwise the precipitated structures created during polymerization redissolved upon cooling. A possible explanation could be the excess of surface charges on the nuclei. It could prevent their growth during polymerization due to high electrostatic repulsion between the growing chain and the surface. However, the goal of the present work was not to explore in-depth the polymerization mechanisms, therefore the preparation conditions were adapted to yield particles obtained in comparable conditions. The amount of KPS was thus decreased to $0.5 \mathrm{mM}$ for the whole series concerning single monomers (route 2).

In such conditions, a range of monodispersed particles from different oligo(ethylene glycol) chain lengths were obtained: 8 different compositions in the number of EO units and 1 with a different cross-linker ratio ( $1 \mathrm{~mol} \%$ instead of $2 \mathrm{~mol} \%$ ). Table 1 summarizes the microgels compositions. In order to facilitate the reading, the average number of EO units of copolymer microgels is given for each composition, using the following formula: $E O n=(1-x) * 2+$ $(x * 8.5)$. This will also help establishing comparison between microgels made of copolymers (route 1) and those made of homopolymers (route 2), where EOn $=n$. At the end of the synthesis, the microgels were purified by several centrifugation-redispersion cycles. The supernatant extracted from the first cycle contained mainly the hydrosoluble species. The 
fraction of water soluble polymers (WSP) was determined by analyzing its dried mass which was compared to that of the mixture after polymerization. As seen in Table 1, the fraction of WSP is quite high for all the samples, in line with literature data [57]. Additionally, it increases by increasing the amount of more hydrophilic monomers (longer EO chains) (Fig. S1). This trend is similar whatever the synthesis route and thus, whatever the KPS content. The orders of magnitude depend mainly on the average number of EO units. 
Table 1. Composition of the synthesized microgels and result of the synthesis

\begin{tabular}{|c|c|c|c|c|c|c|c|}
\hline \multirow[b]{2}{*}{ Name } & \multicolumn{4}{|c|}{ Monomers (mM) } & \multirow{2}{*}{$\begin{array}{c}\text { Average } \\
\text { number } \\
\text { of EO } \\
\text { units } \\
\text { EOn }\end{array}$} & \multirow{2}{*}{$\begin{array}{c}\text { Cross- } \\
\text { linker } \\
(\mathrm{mol} \%) \\
\text { OEDA }\end{array}$} & \multirow{2}{*}{$\begin{array}{c}\text { Fraction of } \\
\text { water- } \\
\text { soluble } \\
\text { species } \\
(\mathrm{wt} \%)\end{array}$} \\
\hline & OEMA 2 & OEMA 3 & OEMA 4 & OЕMА 8 & & & \\
\hline $\begin{array}{c}\text { pOEMA } 2 / 8 \\
(5 \%)\end{array}$ & 90.25 & - & - & 4.75 & 2.3 & 2 & $39 \pm 2$ \\
\hline $\begin{array}{c}\text { POEMA } 2 / 8 \\
(10 \%)\end{array}$ & 85.5 & - & - & 9.5 & 2.6 & 2 & n.a. \\
\hline $\begin{array}{c}\text { pOEMA } 2 / 8 \\
(10 \%, 1 \text { mol\% } \\
\text { EGDA) }\end{array}$ & 85.5 & - & - & 9.5 & 2.6 & 1 & $58 \pm 3$ \\
\hline $\begin{array}{c}\text { POEMA } 2 / 8 \\
(15 \%)\end{array}$ & 80.75 & - & - & 14.25 & 3 & 2 & $53 \pm 3$ \\
\hline $\begin{array}{c}\text { POEMA } 2 / 8 \\
(20 \%)\end{array}$ & 76 & - & - & 19 & 3.3 & 2 & $54 \pm 3$ \\
\hline $\begin{array}{c}\text { POEMA 2/8 } \\
(25 \%) \\
\end{array}$ & 71.25 & - & - & 23.75 & 3.6 & 2 & $68 \pm 3$ \\
\hline рОЕМА 2 & 100 & - & - & - & 2 & 2 & $21 \pm 2$ \\
\hline рОЕМА 3 & - & 100 & - & - & 3 & 2 & $50 \pm 3$ \\
\hline pОЕМА 4 & - & - & 100 & - & 4 & 2 & $67 \pm 3$ \\
\hline
\end{tabular}

\subsection{Size and swelling properties of the microgels}

All the microgels were monodisperse, as shown by their low PDI obtained in DLS. They were also thermoresponsive with different VPTT (Fig. 3 and Fig. S2). Their size increases as a function of the hydrophilic character, both in the swollen state and in the collapsed state, the latter being the size fixed by the polymerization conditions. This trend is observed whatever the synthesis route. A similar trend was also observed previously in the case of OEMA2 and 8 copolymers, using EGDMA as a cross-linker[54]. To date, there is no clear explanation about it. It would require a detailed mechanistic study of the polymerization process to answer this question. 


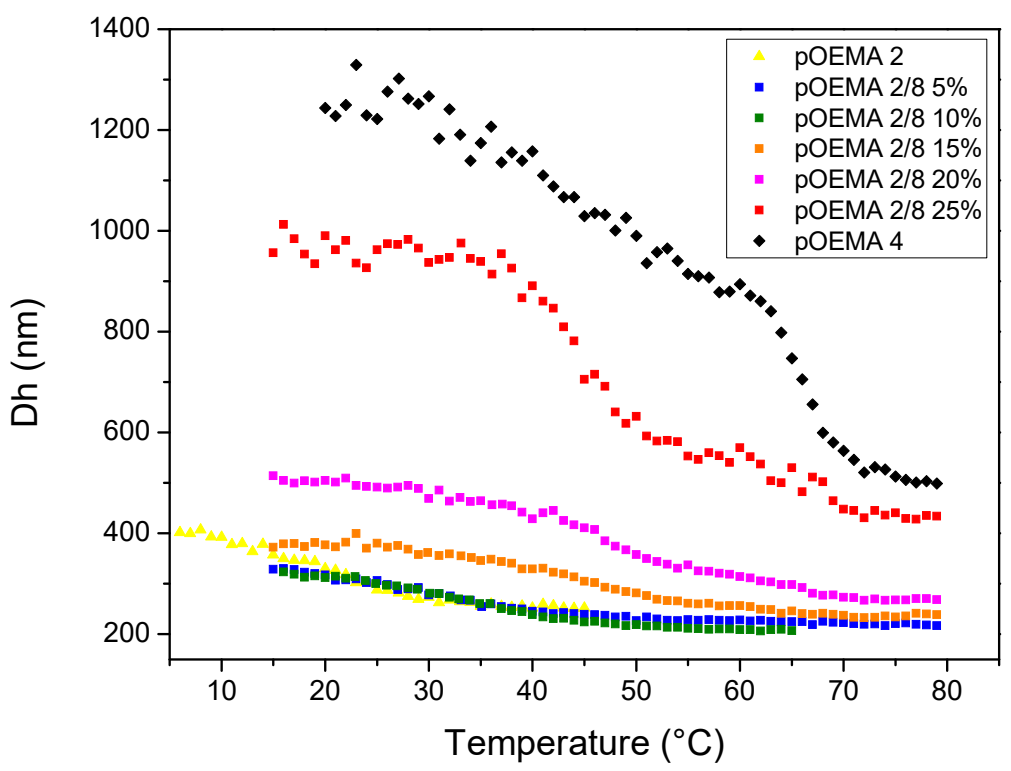

Figure 3. Evolution of the hydrodynamic diameter of the microgels as function of temperature for various the pOEMA microgels. An enlargement is available in Figure S2.

We now discuss the swelling properties and responsiveness of the microgels. Fig. S3 shows the hydrodynamic diameter, normalized by its value in the collapsed state, as a function of temperature for all the microgels. All the microgels display a volume phase transition, from the swollen to the collapsed state, which is defined as the state where the microgels reach a plateau. The temperature at the start of the plateau increases as the length of the EO chains increases, as expected by the increase of hydrophilicity. The VPTTs should be determined at the inflection point of the swelling curves. However, their precise determination was difficult because of the large width of the phase transition. The width of the phase transition increases as the hydrophobic contribution increases. A steeper deswelling is observed for microgels having the highest VPTT. This tendency is observed for both homopolymers and copolymers: the shape of the curves is similar for systems having close VPTT (Fig. S3). 
The swelling ratio is defined as the ratio of the volume in the swollen state to the volume in the collapsed state: $\alpha=\left(\frac{d_{H, 20^{\circ} \mathrm{C}}}{d_{H, T>V}}\right)^{3}$. It increases as the EO chain length increases. This result is in qualitative agreement with previous findings regarding copolymer microgels based on EGDMA cross-linker [54]. Here it is also extended to homopolymer microgels. Furthermore, the range of swelling ratio, from 3 to 16 , is broader. This can be explained by the more hydrophilic nature of the cross-linker and also by its lower concentration. For a given EO composition, the samples with two different cross-linker to monomer ratios have similar plateau temperatures but, as expected, the swelling degree is much higher for the less cross-linked sample (10.2 instead of 3.4) (Fig. S4).

To determine more precisely the temperature at which the fully deswollen state is reached, the behavior of the particles in the brine solution $(0.1 \mathrm{M} \mathrm{NaCl})$ was studied. The idea was to differentiate the responsiveness of the microgels by measuring the onset of flocculation, which takes place only when the unswollen state is reached. It was shown previously that the presence of $\mathrm{NaCl}$ up to $0.1 \mathrm{M}$ induces a negligible shift of the LCST in thermoresponsive microgels (less than $1^{\circ} \mathrm{C}$ ) $[65,66]$, which was further confirmed for pOEMA linear polymers[46]. However, the presence of salt has a significant role on the colloidal stability of microgel suspensions. At low temperatures, suspensions of thermoresponsive microgels such as pNIPAM are colloidally stable when the microgels are in the swollen state, due to negligible Van der Waals attractions. Upon temperature increase, the charge density of microgels increases upon deswelling. In brine solution, where electrostatic repulsions are screened, they flocculate as soon as they reached the collapsed state, because the Van der Waals attractions overcome electrostatic repulsions $[66,67]$. However, when pNIPAM microgels are in the collapsed state but composed of copolymers remaining in good solvent in the shell, such as PEG chains, these chains act as steric stabilizers and prevent flocculation [68, 69]. pOEMA microgels are also negatively 
charged owing to the presence of sulfate groups arising from the persulfate initiator [55]. In the presence of $0.1 \mathrm{M} \mathrm{NaCl}$, it is observed that pOEMA microgel suspensions are stable at low temperature, when the microgels remain swollen, but they all flocculate at a given temperature (Fig. 4A). From this observation, we can conclude that microgels flocculate once they reach the collapsed state. The onset of flocculation is plotted as a function of average number of ethylene oxide units (Fig. 4B). It follows a linear behavior, showing the perfect correlation between the hydrophilic character and the flocculation temperature. Moreover, the flocculation temperature is compared to the LCST values found in the literature for linear polymers prepared by controlled radical polymerization (Fig. S5). A quite nice agreement is found between the two sets of data. It confirms that the presence of $\mathrm{NaCl}$ has only an impact on the flocculation but it does not shift the phase transition towards lower temperatures. Moreover, the occurrence of flocculation demonstrates that attractive forces are not compensated by repulsive steric ones. This means that all the polymer chains are in a bad solvent, even those located at the surface of the particles.

In this section, it was demonstrated that the flocculation temperature is a more precise measurement of the thermoresponsive character of the microgels. From now on, it will be used instead of the VPTT to describe the hydrophilic character of the microgels. Table 2 summarizes the characteristics of each microgel. 


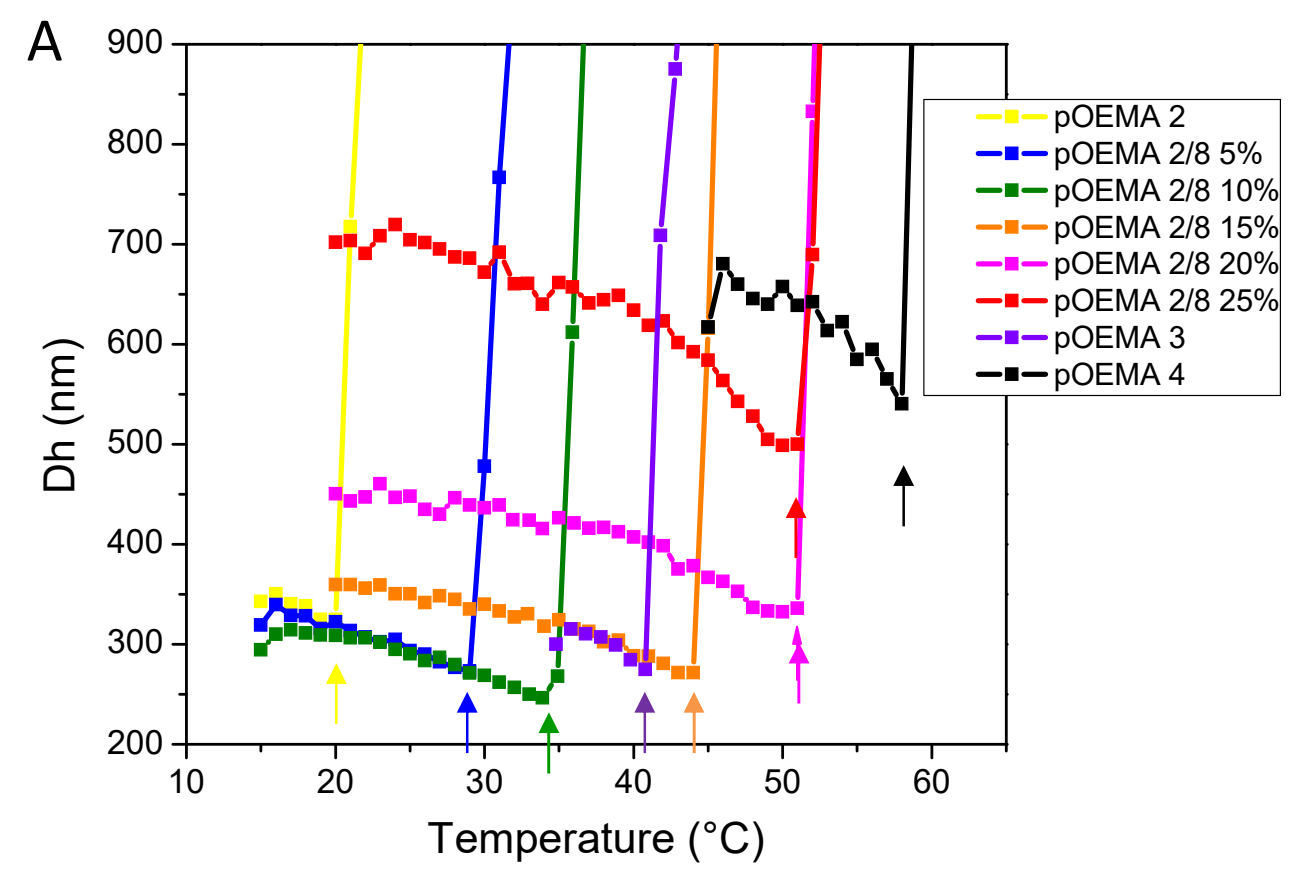

B

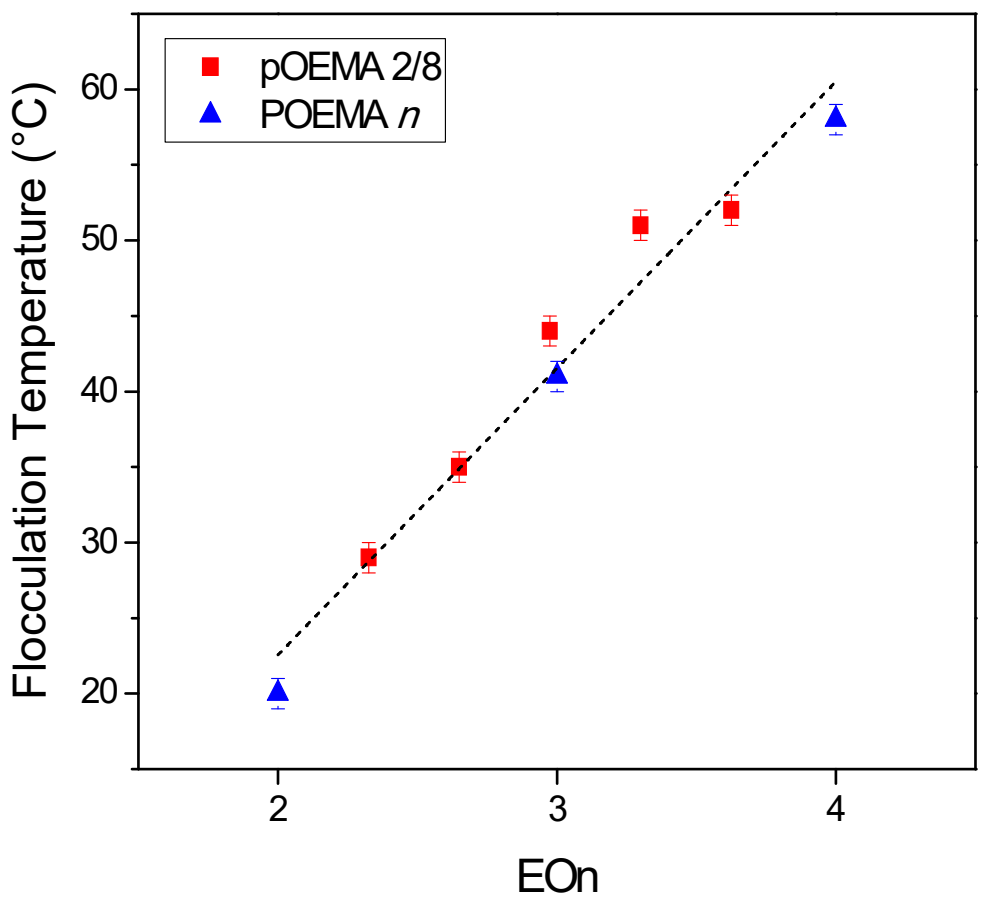


Figure 4. A) Onset of flocculation in $0.1 \mathrm{M} \mathrm{NaCl}$, assessed by DLS measurement; B) Plot of the flocculation temperature as a function of EO number. The dotted line corresponds to the following equation: $\mathrm{T}=-15.4+19 \mathrm{EOn}$. It is the best fit to linear dependency.

Table 2. Summary of microgels properties in aqueous solution.

\begin{tabular}{|c|c|c|c|c|c|}
\hline Microgels & $\begin{array}{c}\mathbf{d}_{\mathbf{h}^{20}}{ }^{\circ} \mathbf{C}(\mathbf{n m}) \\
P D I\end{array}$ & $\begin{array}{c}\mathbf{d}_{\mathbf{h}} \text { plateau } \\
(\mathbf{n m})\end{array}$ & $\begin{array}{c}\text { Swelling } \\
\text { ratio } \alpha\end{array}$ & $\begin{array}{c}\mathbf{T}_{\text {flocculation }} \\
\left({ }^{\circ} \mathbf{C}\right)\end{array}$ & $\begin{array}{l}\text { Theoretical } \\
\text { LCST }\left({ }^{\circ} \mathrm{C}\right)\end{array}$ \\
\hline pOEMA $2 / 8(5 \%)$ & $\begin{array}{l}318 \\
0.03\end{array}$ & 220 & 3.0 & 29 & 33 \\
\hline $\begin{array}{l}\text { pOEMA } 2 / 8(10 \%) \\
\text { - OEDA } 2 \text { mol } \%\end{array}$ & $\begin{array}{l}315 \\
0.02\end{array}$ & 210 & 3.4 & 35 & 39 \\
\hline $\begin{array}{l}\text { pOEMA } 2 / 8(10 \%) \\
\text { - OEDA } 1 \text { mol\% }\end{array}$ & $\begin{array}{l}390 \\
0.04\end{array}$ & 180 & 10.2 & 32 & 39 \\
\hline pOEMA $2 / 8(15 \%)$ & $\begin{array}{l}377 \\
0.01\end{array}$ & 240 & 3.9 & 44 & 44 \\
\hline pOEMA 2/8 (20\%) & $\begin{array}{l}505 \\
0.04 \\
\end{array}$ & 270 & 6.5 & 51 & 49 \\
\hline pOEMA $2 / 8(25 \%)$ & $\begin{array}{l}990 \\
0.02 \\
\end{array}$ & 450 & 10.7 & 52 & 54 \\
\hline pOEMA 2 & $\begin{array}{l}330 \\
0.07\end{array}$ & 250 & 2.3 & 20 & 22 \\
\hline pOEMA 3 & $\begin{array}{l}340 \\
0.10\end{array}$ & 190 & 5.7 & 41 & 52 \\
\hline pOEMA 4 & $\begin{array}{l}1250 \\
0.06\end{array}$ & 490 & 16.6 & 58 & 61 \\
\hline
\end{tabular}

\subsection{Morphology/deformability of the microgels}

In order to learn more about the morphology and deformability of the microgels, AFM and TEM imaging techniques were used. Dilute dried microgels were observed on a substrate. Microgels are thus observed as more or less flattened objects [23, 70]. In TEM, the substrate was a carbon-coated copper grid and in AFM, the substrate was a silicon wafer. These techniques are complementary. In TEM, the inner structure of the microgels may be viewed in transmission whereas AFM allows recording the thickness of the sample (profile view).

TEM views were recorded for all the samples (Fig. 5A and Fig. S6). Highly monodispersed samples were obtained for all the homopolymers. However, those with the 
shortest EO chains have a round and smooth structure, whereas pOEMA 4 exhibits a rough surface with digitations, typical of soft particles. Regarding the copolymers, all the particles look homogeneous in size but their shape evolves from round-shaped particles for the lowest OEMA8 content to less spherical, rough and digitated structures when the OEMA8 content increases. Increasing the hydrophilicity of microgels seems to increase the softness of microgels.

This trend is confirmed by AFM (Fig. 5 and Fig. S7). The loss of the round shape upon hydrophilicity increase is confirmed. Furthermore, analysis of the heights revealed that they were much smaller than the in-plane diameter. The microgels were thus in a flattened conformation. Fig. 5B shows the superposition of the height profiles for the various copolymer microgels. The height of microgels decreases as the content of EO units increases. At the same time, the in-plane diameter increases, in accordance with the evolution of the diameter of the particles in the collapsed state. To quantify the deformation of the microgel on the solid substrate, the ratio between the height and the full width at half maximum ratio (FWHM) was calculated (Fig. 5C and Table S8). A ratio close to 1 means that particles are poorly deformable. It varied between 0.4 and 0.1 , and decreased linearly as the flocculation temperature increased. This means that increasing the EO content increases the deformability of the particles. This statement remains valid for both homopolymers and copolymers.

In summary, a range of pOEMA microgels with various VPTT was prepared, whose deformability increases as their hydrophilicity increases. 

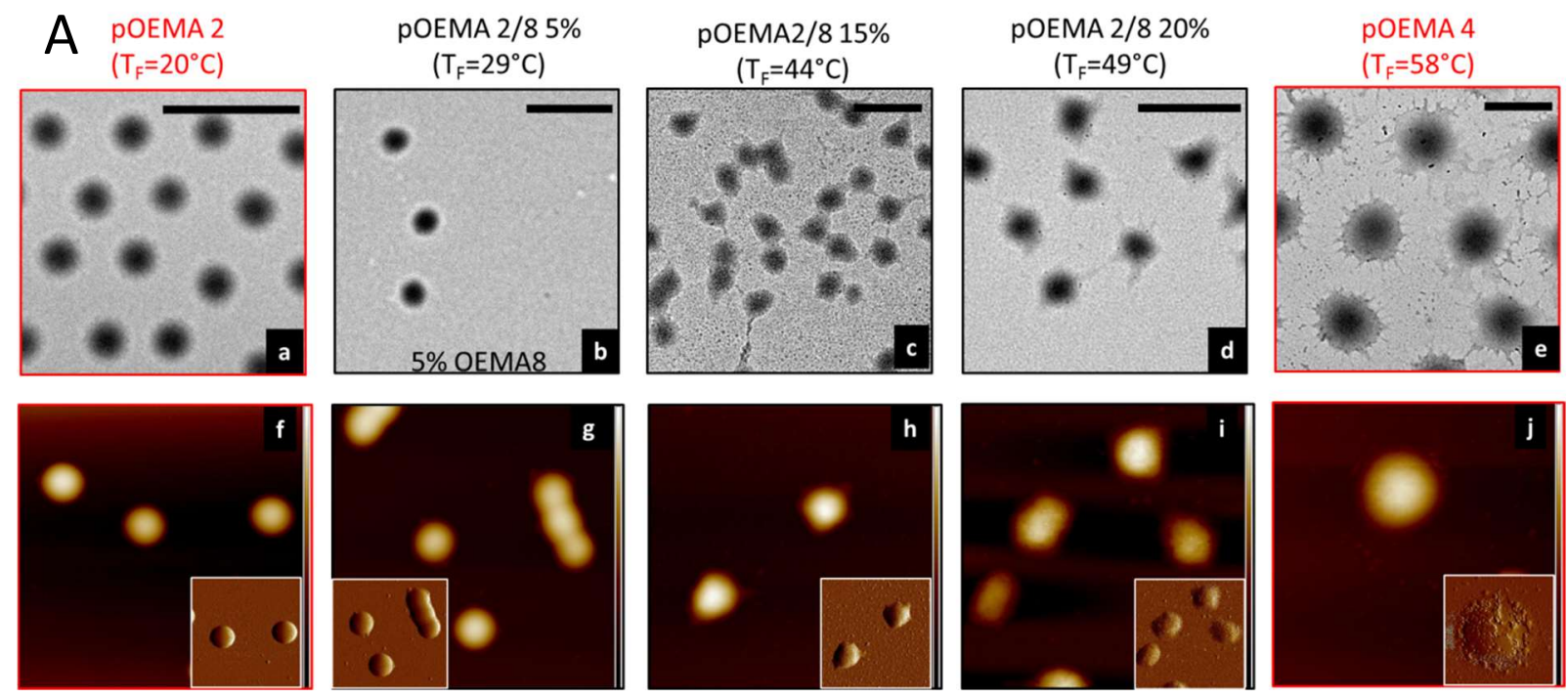

B
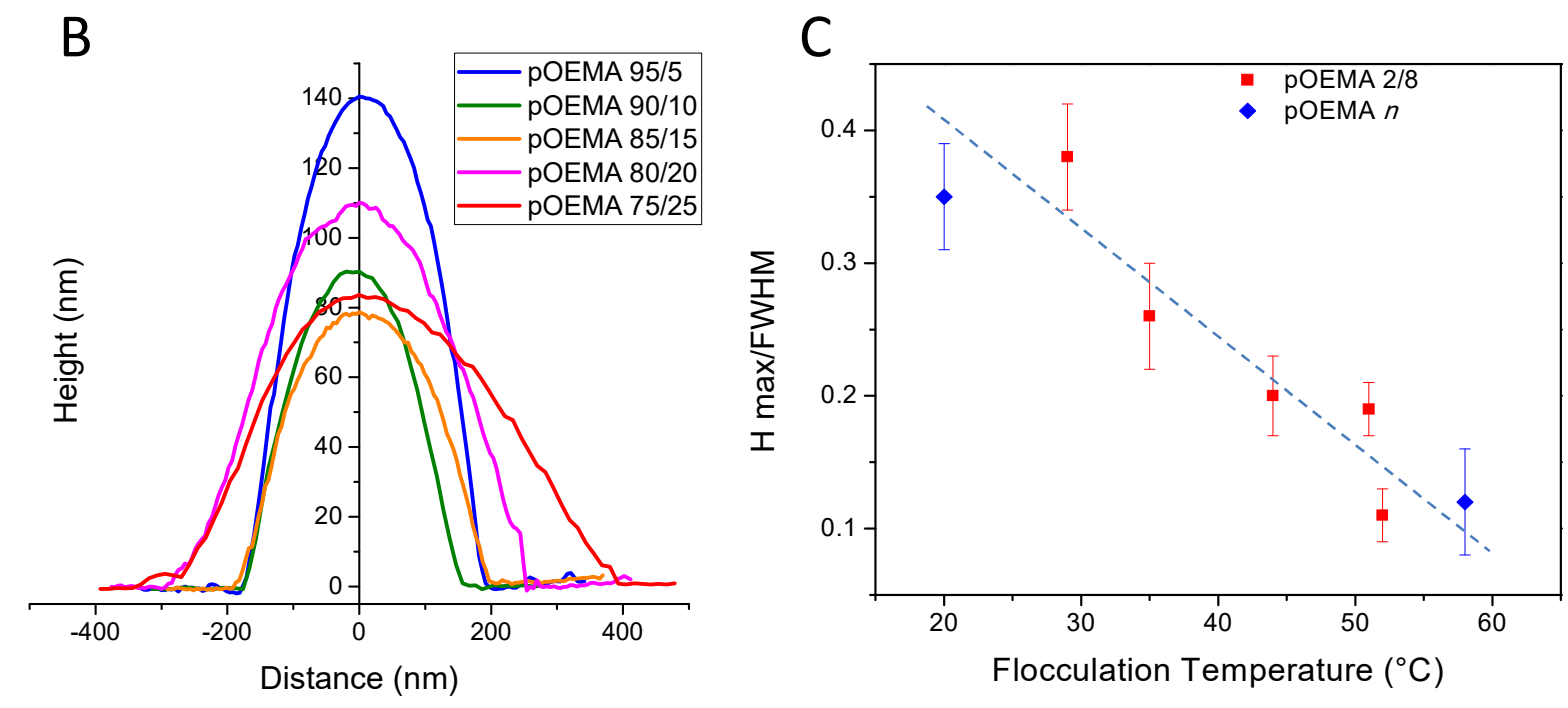

Figure 5. TEM and AFM characterizations of some selected dried microgels. A. TEM images (a-e) and AFM images showing height views and phase images insets (f-j) for samples: pOEMA 2 (a; f), pOEMA 2/8 5\% (b; g), 15\% (c ; h), 20\% (d ; i), pOEMA 4 (e ; j). Profiles of the pOEMA 2/8 dried microgels after deposition on silicon wafer (B) and evolution of the ratio between the maximal height and the width at half height as a function of thermoresponsiveness (C). The dotted line is a guide for the eye to evidence the linear variation of the deformation with $\mathrm{T}_{\text {flocculation. }}$ 


\subsection{Behavior of the microgels at a liquid interface (air/water)}

The goal of the study is to understand the behavior of microgel at the oil-water interface. In a previous study, it was demonstrated that the features of oil-water and air-water interfaces obtained by the pendant drop method were very similar [34]. In the present work, only interfacial properties of the pOEMA microgels at the liquid-air interface are presented. In this technique, microgels are initially dispersed in an aqueous drop and their spontaneous adsorption at the air-water interface is observed by measuring the evolution of the surface tension as a function of time. One can thus determine the kinetics of adsorption, as well as the surface tension decrease after a given time. This kind of studies was previously conducted on pNIPAM microgels[34], showing that their adsorption was a cooperative and irreversible phenomenon, leading to the same surface tension at infinite time, whatever the microstructure of the pNIPAM microgels, after a concentration threshold has been overpassed. Conversely, their adsorption kinetics was highly dependent on their concentration, their deformability/microstructure and electrostatics. The present study is intended to study the role of the composition and structure of the pOEMA microgels on their adsorption. The microgel concentration was thus kept constant and equal to $0.05 \mathrm{wt} \%$. In order to remove electrostatic contribution, a small amount of $\mathrm{NaCl}\left(10^{-2} \mathrm{M}\right)$ was added to the aqueous phase. It should also be underlined that all the microgels were carefully washed by repeated centrifugations-redispersion cycles, in order to extract WSP. Indeed, the presence of WSP has drastic effects on the adsorption kinetics [34].

All the prepared microgels were found to adsorb at the air-water interface, with a profile similar to that of pNIPAM (Fig. 6A). The surface tension is that of water at short times, then it decreases and reaches a plateau. The value of the plateau (surface tension at $10000 \mathrm{~s}$ ) gives the ability of the microgel to decrease interfacial energy. In Fig. 6B, it is plotted as a function of flocculation temperature, or equivalently of the average number of EO units. The reduction of the surface tension is all the more pronounced since the microgels are hydrophobic. This 
evolution is not regular, but there is a visible tendency to decrease more the surface tension as the microgel becomes more hydrophobic. The characteristic time of adsorption $t^{*}$ is generally defined as the time required to decrease the surface tension by half of its final reduction rate. In our case, it should be the time required to reach $60 \mathrm{mN} / \mathrm{m}$. In the case of pNIPAM microgels, the adsorption kinetics was all the faster since the microgels were soft and deformable, probably due to the presence of dangling chains. In the case of pOEMA, the characteristic times were plotted as a function of flocculation temperature (Fig. S10). This graph does not show any clear tendency, except that those being the most hydrophobic (pOEMA 2, pOEMA 2/8-5\%), having a flocculation temperature below or close to the experimental temperature $\left(22^{\circ} \mathrm{C}\right)$, are much slower than the others. This result is consistent with the fact that the more hydrophobic microgels are in a deswollen state, probably without dangling chains, and thus have a more rigid structure, which slows down their adsorption kinetics. Again, the study shows that the size of the microgels is not an important factor. Indeed, the microgel of pOEMA $2 / 8$ containing $5 \%$ of OEMA 8 , is smaller than the sample containing $25 \%$ of OEMA 8 , but it has a slower kinetics. It confirms that diffusion does not govern adsorption.

In the case of pOEMA 4, which was found to be very soft and deformable, a faster dynamics would be expected. Its adsorption time is longer than all other microgels, showing an atypical behavior.

The role of the cross-linking ratio confirms the role of microgel deformability on the adsorption. Indeed, as shown in Fig. S9, the softer microgels obtained by decreasing the cross-linking ratio, adsorb faster than the ones which are more cross-linked by one order of magnitude ( $20 \mathrm{~s}$ instead of $200 \mathrm{~s})$. 

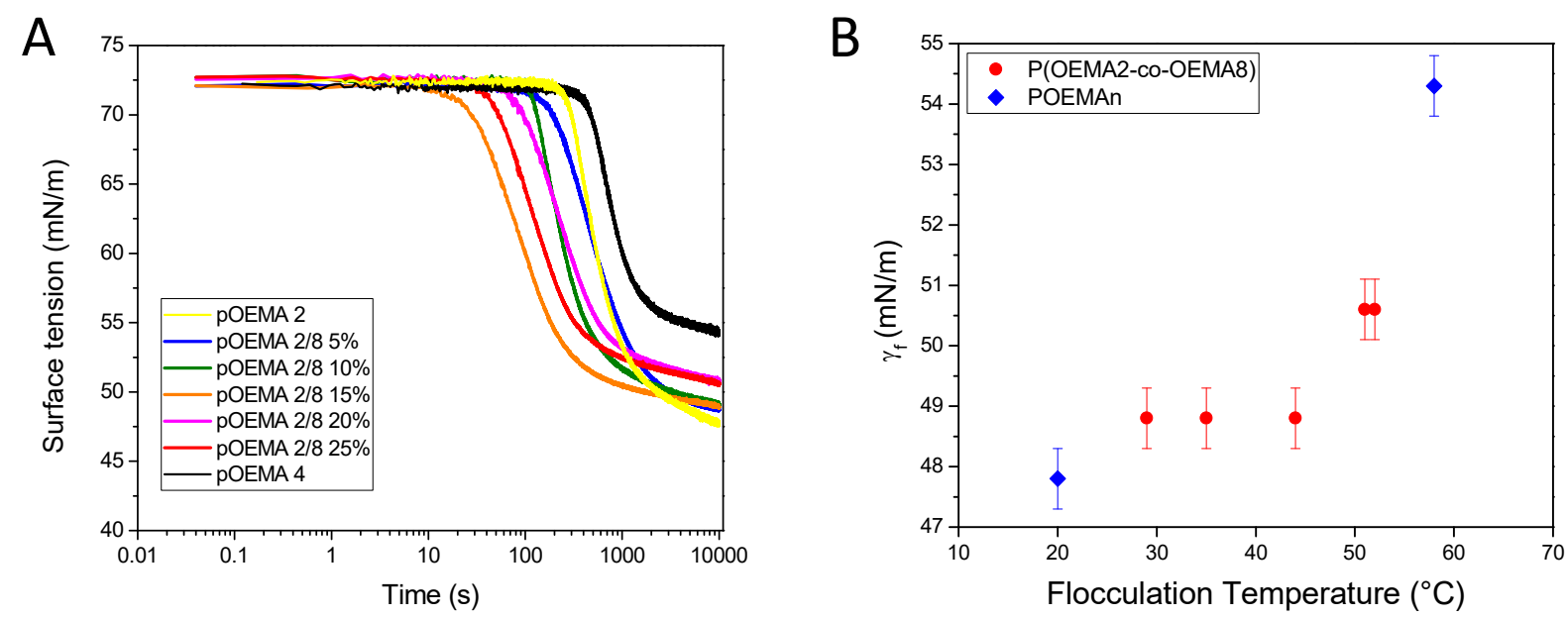

Figure 6. Dynamic surface tension of the various microgels (A) and evolution of the final surface tension as a function of flocculation temperature of the corresponding microgels (B). 


\subsection{Properties of the emulsions stabilized by the microgels}

\subsubsection{At ambient temperature}

Finally, the pOEMA microgels were used as emulsion stabilizers. Oil-in-water emulsions were prepared by mixing $30 \mathrm{wt} \%$ of dodecane with an aqueous solution containing a fixed amount of microgels $(0.05 \mathrm{wt} \%)$. All the emulsions were prepared using an ultra-turrax, operated for $30 \mathrm{~s}$ at $4^{\circ} \mathrm{C}$. This temperature was chosen well-below the VPTT of all the microgels, in order to promote their adsorption in the swollen state. Emulsion properties were then studied at $22^{\circ} \mathrm{C}$. Results are presented in Fig. 7 and Fig. S11. Stable oil-in-water emulsions were successfully obtained with all the microgels, except with pOEMA 2, whose VPTT is below ambient temperature. In this case, the emulsion destabilizes immediately after preparation when the emulsion is at $22^{\circ} \mathrm{C}$. All the others presented large drops in the range $10-100 \mu \mathrm{m}$, which creamed rapidly after preparation (with few seconds). The size range is typical for Pickering emulsions prepared with very low amounts of particles, where the interfacial area between oil and water is solely determined by the surface area that can be covered by the particles. This regime is called the limited coalescence process [62] and will be discussed later.

Regarding their dispersion state, it is noticed that emulsions prepared with high VPTT microgels are less flocculated than those with low VPTT ones. This is visible both on the macroscopic images where the limit between the creamed layer and the subnatant is not smooth, and on microscopy images, where drops form clusters and are more heterogeneous in size. It was shown in previous studies that the flocculation state of emulsions-stabilized by microgels results from bridging between drops, where microgels can adsorb simultaneously two adjacent drops [71]. In this case, drops are adhesive and separated by large flat films. The adhesion angle, observed at the junction with the free interfaces, is all the higher since adhesion is stronger [71]. Bridging is generally promoted by the less deformable microgels, which slowly adsorb and 
deform, thus protruding at the interface. In the case of pOEMA microgels, the occurrence of bridging was also confirmed by observing the flowing properties of emulsions (Fig. 8): bridged emulsions lead to plug-flow behavior, a behavior more pronounced for the more hydrophobic microgels, which are also the most rigid ones, thus confirming this tendency. Furthermore, the comparison between microgels having two different cross-linking ratios shows that the less cross-linked, and thus the most deformable, lead to the less flocculated emulsion.

Regarding their stability, all the emulsions were stable at rest, but it was noticed that those prepared with the low VPTT microgels, i.e. the most rigid ones, were mechanically fragile. They underwent coalescence upon gentle shaking. This observation is in agreement with previous findings concerning pNIPAM microgels [2].

In summary of this part, the properties of pOEMA-stabilized emulsions (flocculation state, stability) are related to microgel deformability, which is itself the consequence of microgel composition.

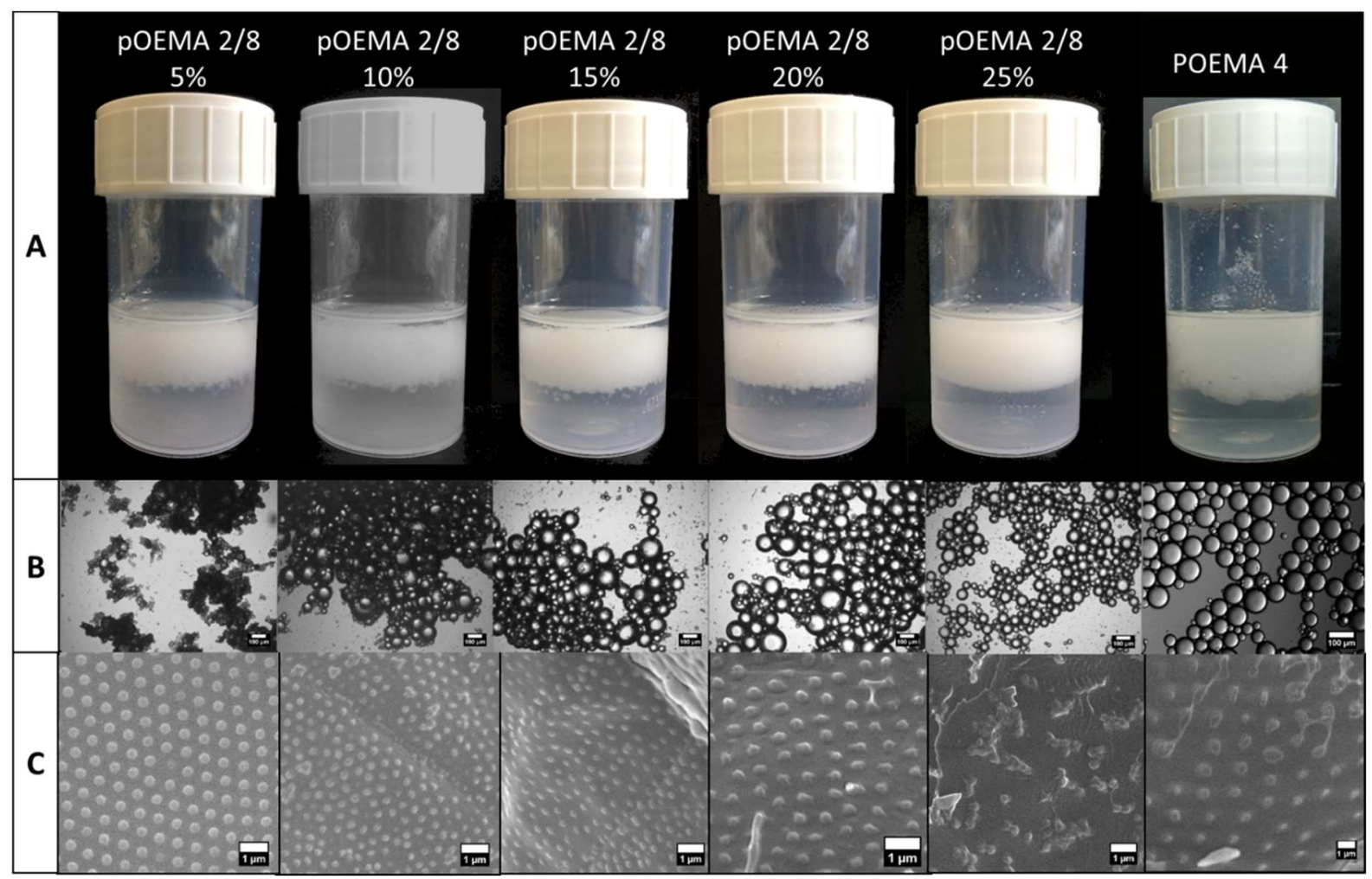


Figure 7. General view of the dodecane-in-water emulsions obtained with $0.05 \mathrm{wt} \%$ of microgels, at $22^{\circ} \mathrm{C}$. The dodecane fraction is $30 \mathrm{wt} \%$. Row A shows the macroscopic views, row B shows optical microscopy images of the drops (scale bar is $100 \mu \mathrm{m}$ ), row $\mathrm{C}$ shows the cryoSEM images of the microgels at the drop surface (scale bar is $1 \mu \mathrm{m}$ ).

\subsubsection{Organization of microgels at the interface}

The organization of microgels at the drop surface can be investigated by two complementary methods: 1) at the local level, scanning electron microscopy in cryogenic conditions allows visualizing the drop surface[2], 2) the determination of the drop interfacial area as a function of microgel number, in the so-called limited coalescence regime, gives a bulk quantitative approach to measure the surface coverage [2]. The surface coverage $C$ is defined as the ratio between the equatorial area of the particles in solution (undeformed) and the total interfacial area (see SI, eq. 2). Fig. 7C and Fig. S12 gather the cryoSEM views of the drops covered by the different pOEMA microgels. In the case of pOEMA $2 / 85 \%$ and $10 \%$, large adhesion angles between the drops are observed, which confirm the occurrence of bridging. Regarding the organization of microgels at the surface of drops, for most of the samples, the drops were covered by a uniform layer of regularly-spaced microgels. The only exception was pOEMA 2/8 $25 \%$, which was organized into clusters. At the opposite, pOEMA 2/8 5\% exhibited the most perfect hexagonal organization, made of spherical protuberant particles. Upon increasing the EO content, the degree of organization decreased and the microgels appear as less spherical particles, being even flattened. In attempt to quantify microgel organization and deformation, the lattice parameters, in other words the center-to-center distances between microgels $d_{C C}$, were extracted from the data (Table 3). In few cases, two lattice parameters were found, depending on the investigated area. In all cases, $d_{C C}$ was bigger than the initial diameter in 
solution, meaning that the microgels are flattened at the surface. The surface coverage, calculated using the following formula:

$C_{\text {CryoSEM }}=\frac{\pi d_{H}^{2}}{2 \sqrt{3}-d_{C C^{2}}}$

is reported in Table 3. In the case of a monolayer of spherical particles in a hexagonal array, the surface coverage is $90 \%$. Our data are much lower than this limit because the area of the non-deformed microgel is smaller than the effective covered area.

The limited coalescence process was used as a complementary approach to determine the microgel organization. The principle is the following: emulsions are prepared with very low amounts of microgels, where all the microgels adsorb irreversibly at the interface. If the surface area of created droplets is high, the droplets undergo coalescence to decrease the interfacial area, until the coverage is sufficiently dense to stop coalescence. It generally leads to a uniform drop size (see SI S13 for details). Therefore, the final size of emulsion drops is a function of the number of microgels and of their organization. The size of emulsions was thus measured as a function of microgel concentration for four pOEMA microgels: pOEMA 20\%, pOEMA 25\%, pOEMA 4 and pOEMA $10 \%$ with 1 mol\% of cross-linker. Samples leading to highly bridged emulsions were discarded because all the emulsions were polydisperse. For the four investigated samples, emulsions were monodispersed in the investigated range of microgel concentration. The inverse of the diameter was plotted as a function of microgel number normalized by the dispersed phase volume, or, equivalently the surface area of the nondeformed particles (Fig. S14). In all cases, a linear relationship was obtained, which confirms the occurrence of the limited coalescence phenomenon. Moreover, the slope of the line is $\frac{1}{6 C}$. This calculation uses an assumption for the evaluation of the number of microgels. Indeed, the amount of water that remains in the collapsed state of the microgels is needed (eq. 3 in SI13). 
The results of the calculation are in quite good agreement with surface coverages obtained by cryoSEM. In any case, the obtained surface coverages are below $90 \%$, meaning that either the microgels are apart from each other leading to poorly covered drops or the microgels are deformed and the microgel diameter to be considered is larger than the hydrodynamic diameter measured in the suspension. As for pNIPAM microgels, this last explanation is valid as the microgels are in contact as proven by the CryoSEM pictures.

In conclusion of this section, the interfacial organization of microgels at drop surface shows that the microgels are deformed at the interface. However, in opposition to pNIPAM microgels where only the cross-liking density was varied, there is no clear correlation between the level of deformation and the softness of the microgels. The only conclusion is that the more rigid microgels lead to the best level of ordering.

Table 3. Organization of microgels at the drop surface, determined by cryoSEM or by the limited coalescence process (see SI13).

\begin{tabular}{ccccc}
\hline Microgel & $\begin{array}{c}\mathrm{D}_{\mathrm{H}}(\mathrm{nm}) \\
\text { In water, at } \\
20^{\circ} \mathrm{C}\end{array}$ & $\mathrm{d}_{\mathrm{cc}}{ }^{\text {CryoSEM }}(\mathrm{nm})$ & $\mathrm{C}_{\text {Cryo-SEM }(\%)}$ & $\mathrm{C}_{\mathrm{LC}}(\%)$ \\
\hline OEMA95/5 & 318 & $715 \pm 30$ & $20 \pm 4$ & \\
& & $580 \pm 50$ & $27 \pm 5$ & $/$ \\
OEMA90/10 & 315 & $450 \pm 40$ & $44 \pm 7$ & \\
OEMA90/10 & 390 & $660 \pm 50$ & $28 \pm 3$ & $26 \pm 5$ \\
(OEDA 1\%mol) & & $530 \pm 80$ & $45 \pm 9$ & $/$ \\
OEMA85/15 & 377 & &
\end{tabular}




\begin{tabular}{ccccc} 
OEMA80/20 & 505 & $600 \pm 60$ & $64 \pm 9$ \\
& & $700 \pm 80$ & $47 \pm 9$ & $54 \pm 7$ \\
OEMA75/25 & 990 & $/$ & $/$ & $23 \pm 4$ \\
pOEMA 4 & 1250 & $1800 \pm 130$ & $43 \pm 5$ & $29 \pm 5$ \\
\hline
\end{tabular}

\subsubsection{Thermoresponsive behavior of the emulsions}

Finally, pOEMA microgels constitute a new range of thermoresponsive emulsifiers, with a controlled tunable VPTT. Therefore, emulsion stability was investigated in response to temperature increase at $50^{\circ} \mathrm{C}$, both for homopolymers (Fig. S15) and copolymers (Fig. 8). At this temperature, some of the microgels have reached their collapsed state (pOEMA 10\%, 15\%, pOEMA 3), but pOEMA 25\% and pOEMA 4 should still be partially swollen. The state of the emulsion was controlled macroscopically and the drop size was measured by optical microscopy. At the macroscopic level, after $48 \mathrm{~h}$ of storage, and even after 4 months, most of the emulsions remain stable. No macroscopic phase separation occured. The only one which demulsified is the one stabilized by pOEMA 4, which is still below its VPTT. These results are contradictory to the expected situation, where collapsed microgels should lead to demulsification. These results were double checked in the presence of brine, where the collapse of the microgels was correlated to their flocculation in aqueous solution. Figure S16 confirms the absence of macroscopic phase separation which does not mean the absence of change in the structure of the emulsions. Indeed, the size of the drops showed an increase after temperature raise, which is more pronounced for microgels having the lowest VPTT. A simultaneous evolution of the emulsion texture was observed. The bridging between drops decreased and the emulsion flowed instead of making a plug-flow.

In conclusion of this part, despite the successful preparation of a range of pOEMA microgels with varying VPTT and their ability to stabilize Pickering emulsions, it was demonstrated that 
the achievement of thermoresponsive emulsions with tunable temperature-controlled stability is not straightforward and will be discussed below. 

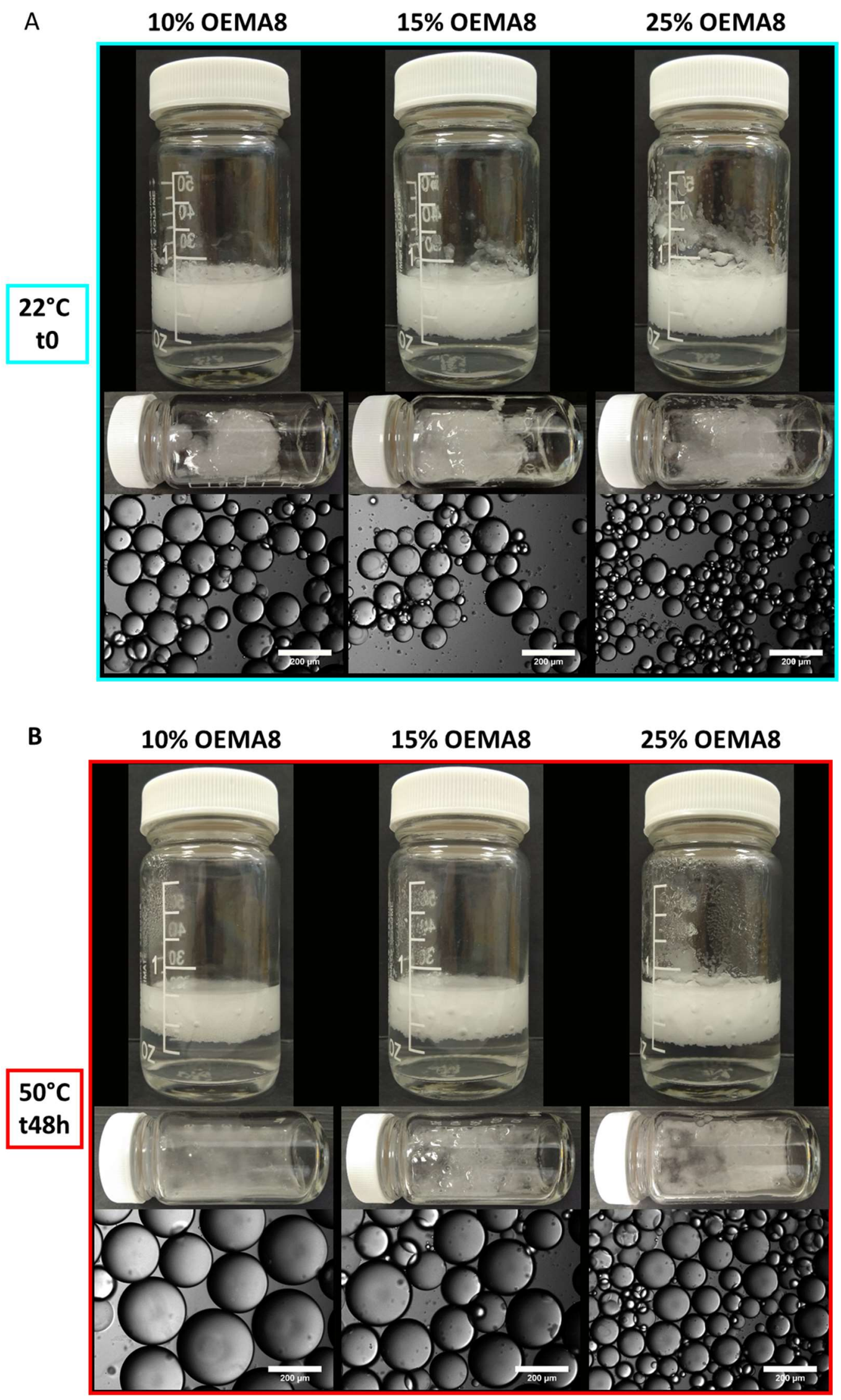
Figure 8. Evolution of the emulsion properties and stability upon increasing temperature. Emulsions stabilized by different microgels: pOEMA $2 / 8$ with 10,15 , and $25 \%$ of OEMA 8 respectively. The micrographs show the emulsion in the quiescent state, after creaming (top row), after tilting the vial (intermediate row) and optical microscopy images of the drops (low row, scale bar is $200 \mu \mathrm{m})$ at $22^{\circ} \mathrm{C}$ and $50^{\circ} \mathrm{C}$.

\section{Discussion}

\subsection{Microgel structure and deformability}

In this work, a series of thermoresponsive microgels with varying VPTT were synthesized, with the intention to relate this parameter to the interfacial properties of microgels and to their emulsification properties. However, establishing simple relations is not straightforward since other important parameters such as the softness and the microstructure influence interfacial properties, as demonstrated in the case of pNIPAM systems. Regarding pOEMA microgels, the abundance of literature should, in principle, help building the structure-property relation. However, small changes in the synthesis conditions of microgels such as surfactant addition, polymerization temperature or the type of cross-linkers, may influence significantly the resulting microstructure, which hinders a full comparison with existing data. In this work, we attempt to draw a general picture of the role of VPTT by following the two-fold polymerization strategy- homo- versus copolymers. The unification of the results appears in Fig. 4B where the average EO number becomes the sole parameter describing the evolution of the VPTT, or equivalent of the flocculation temperature. To the best of our knowledge, such systematic description had not been attempted before. This approach discards a discussion about the microstructure of microgels. The fact that the microgels obey the same laws whatever their synthesis process, will strengthen our conclusions about the role of the number of EO units and thus, about the microgel hydrophilicity. 
As described in the result section, both characterizations, in the bulk phase and at the interface, highlight the evolution of microgel softness as a function of EO unit number or equivalent of the VPTT. In the bulk phase, the swelling ratio increases as the VPTT increases (Table 2). This is in agreement with previous studies obtained with a different cross-linker, which also demonstrated that such microgels consist of a dense core surrounded by a fuzzy shell in the swollen state [54]. At the solid interface, the deformability increases with the VPTT, as shown by the shape of the microgel, the roughness of their contour and by the height-to-width ratio measured by AFM. The quantitative bulk and interfacial parameters are gathered in Figure 9, where the deswelling ratio, defined as $\left(\frac{d_{H, T>V P}}{d_{H, 25^{\circ} \mathrm{C}}}\right)^{3}$ is compared to the flattening in AFM, defined by the maximal height to FWHM ratio. Strikingly, bulk and interfacial data follow the same linear trend as a function of VPTT. It can be concluded unambiguously that microgels with the highest VPTT are the softest and the most deformable.

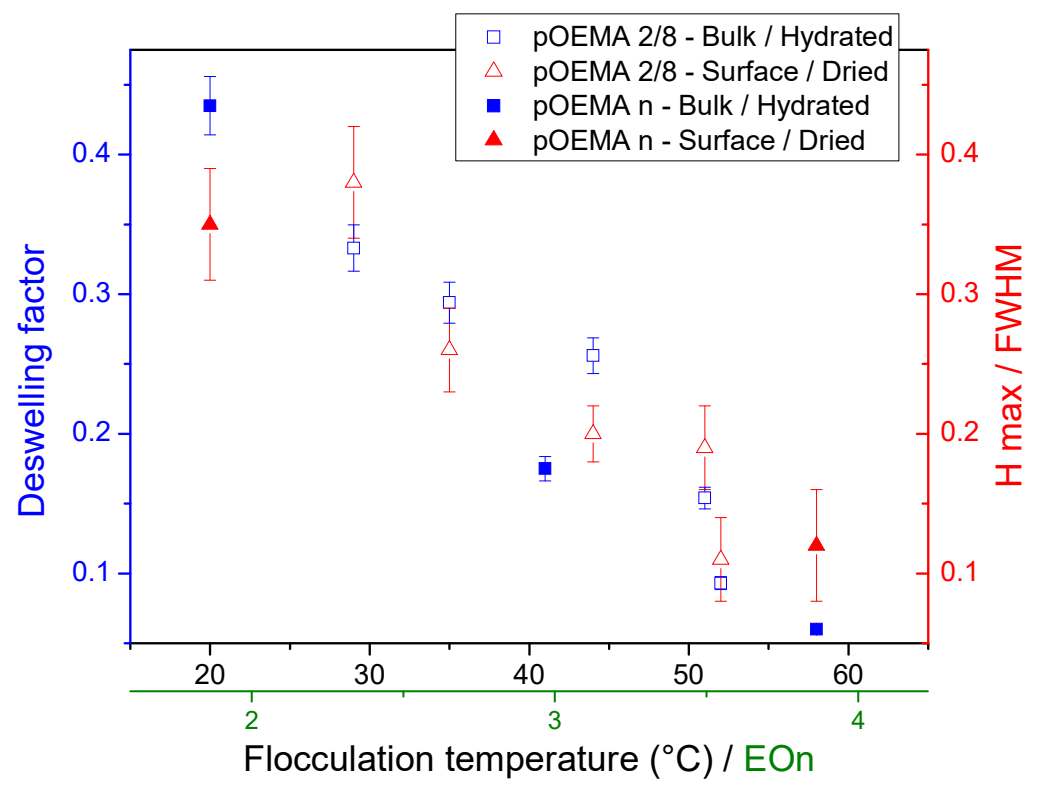

Figure 9. Comparison between the deswelling ratio and the maximal height to FWHM ratio obtained after deposition and drying on silicon substrate, as a function of flocculation temperature. 
In summary of this section, pOEMA microgels with tunable VPTT simultaneously present changes in their structure: 1) increasing size due to increasing thickness of the fuzzy shell, 2) increasing swelling ratio in the bulk phase, 3) increasing flattening at the interface. In the next part, we will discuss the effect of these structural changes, in combination with modification of the hydrophilicity, on their behavior at liquid interfaces. Results will be compared to pNIPAM microgels, whose structure effects are already deciphered.

\subsection{Behavior of pOEMA at liquid interfaces - Comparison with pNIPAM microgels}

This part of the discussion now concerns the spontaneous adsorption of microgels at a flat interface and the stabilization process of emulsions, taken at ambient temperature. Compared to pNIPAM microgels, pOEMA microgels present similar features: they adsorb spontaneously at the liquid interface; the surface tension drops after a given time and reaches a plateau. The kinetics of adsorption seems to be ruled by the softness of the structure, given that the most rigid microgels adsorb slower than the softer ones; the adsorption is not governed by diffusion. Compared to pNIPAM, the main difference is the value of the surface tension at long times, called the final surface tension, which is dependent on the chemical nature of the adsorbed polymer chains. This value depends on the balance between hydrophobic and hydrophilic groups. It increases as the hydrophilicity of the microgel increases and is therefore related to the VPTT. It could be hypothesized that the most hydrophilic polymers adsorb less efficiently to the liquid interface and could eventually possess worse emulsifying properties.

Regarding the emulsifying properties, pOEMA microgels present numerous common features with pNIPAM analogs: when used at temperatures below their VPTT, they serve as Pickering emulsifiers and promote emulsion stability at rest for years; the Pickering effect is demonstrated both by the limited coalescence process and completed by cryoSEM observations of the microgels located at the drop surface; moreover, both types of experiments show that microgels 
are flattened at the drop surface and that the drops are densely covered by the microgels; emulsions prepared with the low VPTT microgels, which are also the most rigid, are flocculated by bridging between drops; such emulsions are mechanically fragile, whereas those stabilized by softer microgels, having a higher VPTT, are more mechanically stable. Their behavior is similar to the one observed with pNIPAM microgels with varying cross-linking density [71]. These features tend to prove that all the thermoresponsive pOEMA microgels are able to efficiently stabilize Pickering emulsions if used in the swollen state. The mechanical stability depends on the state of emulsion bridging. It is the direct consequence of microgel softness and thus related to microgel VPTT. From these observations, it can be concluded that all the thermoresponsive microgels are sufficiently amphiphilic to adsorb at the interface. It means that they should possess a minimal hydrophobicity to promote this adsorption. Above this threshold, hydrophilicity does not strongly influence emulsion properties, which are more sensitive to the microstructure and softness of the microgels.

Considering the high parallel between pNIPAM microgels and pOEMA, the thermal stability of emulsions was expected to follow the evolution of the VPTT. Indeed, until now, all the pNIPAM-stabilized $\mathrm{O} / \mathrm{W}$ emulsions could be destabilized on demand by raising the temperature above the VPTT. Conversely, the phase transition of pOEMA does not affect the macroscopic stability of the emulsion. An increase of the drop size is observed but this coalescence does not propagate to the full sample to induce phase separation. This statement is also true in the presence of $\mathrm{NaCl}$, when the collapse of microgels was evidenced by their flocculation in purely aqueous medium. A few years ago, we reported an example of water-in-oil emulsions that could not be destabilized on demand [72]. It was discovered that the VPTT of the microgels was suppressed because the microgels were partially swollen with the protic oil, here a series of fatty alcohol. In the case of pOEMA microgels, we seek for interactions between the alkanes and the microgels. However, there was no evidence of any uptake of the oil by the microgel 
could and the VPTT remained unchanged even after contact with the oil (results not shown). This lack of destabilization raises the following question: what is really understood about the destabilization mechanism? A few years ago, it was shown that pNIPAM microgel do not desorb at temperature above the VPTT [73]. It was thus believed that microgel collapse could create holes in the interfacial film and disconnect adjacent microgels. It was proved recently that after adsorption at a liquid interface, microgel remain flattened and keep their footprint at the interface, even above their VPTT, while changing their swelling state by adapting their extension in the bulk phase [74]. This kind of experiment was carried out on flat and quiescent monolayers. To reach a complete view of emulsion stability, further investigations would be necessary to understand the coalescence mechanism, which implies understanding the physical properties of the film between two drops: static properties, like the interactions between two monolayers of microgels, and dynamic properties like mechanical response under disturbance as well as hydrodynamics of drainage. In the case of pOEMA microgels, the incomplete coalescence above the VPTT could be explained by a high cohesion of the microgel monolayer. Such cohesion might be supported by connection between adjacent microgels, mediated by the presence of soft shells. Recently, Billon et al. demonstrated the unique properties of some pOEMA microgels that form a self-assembled and self-supported film upon simple evaporation of water [59]. Such films could be rehydrated without losing their integrity, contrarily to pNIPAM ones. This observation indicated that strong attractive interparticle interactions can be achieved, probably resulting from a combination between the microstructure (soft shell, dangling chains) and the chemical nature of the backbone (hydrogen bondings). We speculate that similar effects could explain the remarkable properties of interfacial monolayers. A possible scenario could be the following: after temperature increase above the VPTT, microgels remain adsorb but some coalescence events occur, thereby reducing the total interfacial area. It results in microgel compression, and promotes lateral interpenetration. When the monolayer 
becomes sufficiently cohesive and thus elastic, coalescence stops. Further investigation is required to decipher the mechanism. At the opposite, one of the microgels, pOEMA 4, leads to full destabilization upon temperature raise below the VPTT. There is no clear interpretation about this fact, except that one can notice it is the most hydrophilic. Further investigation should be performed to learn about the microstructure of this microgel.

\section{Conclusion}

This work had the two-fold objective to explore the role of the chemical composition of microgels on their ability to stabilize emulsions, and to extend the scope of applications of such emulsions, by using biocompatible and easy to prepare microgels. POEMA microgels offer an ideal opportunity to fulfill both objectives thanks to the great flexibility of the hydrophilic/hydrophobic balance, at the origin of a fine tuning of the VPTT. The systematic change of the average length of ethylene glycol side chains, achieved by two independent polymerization strategies, reveals the perfect correlation between the VPTT, or equivalently the flocculation temperature, and the EO chain length, independently of the fine microstructure of the microgels. Despite this simple correlation between the chemical composition and the thermoresponsive behavior, the structure of microgels is also the consequence of reactional conditions during polymerization, which affects in particular the distribution of the cross-linker. As a result, the swelling ratio and thus the deformability of microgels increase by increasing the number of EO segments. It has consequences on the behavior of microgels at liquid interfaces. All the microgels can stabilize oil-in-water emulsions when used below their VPTT, but the most deformable ones lead to non-flocculated emulsions, which have the highest mechanical stability. However, when raising the temperature above their VPTT, no demulsification occurs with most of the pOEMA microgels although the drop size increases. 
Only one candidate, which is also the most hydrophilic, fails in stabilizing the emulsion at temperature below its VPTT.

The intent of this work was to set up a bridge between pNIPAM model microgels, for which fundamental principles of emulsion stabilization have been established $[2,3,10,35,36,71,75-77]$, and the growing chemical diversity of the newly developed ones, either synthetic [37,78-81] or biomolecule-based ones [39,40,42,82-84]. A common feature of these particulate emulsifiers is the amphiphilic nature of the polymeric backbone. Through the synthesis of a range of pOEMA microgels with varying amphiphilicity, we were seeking for establishing the role of interfacial tension on the ability of such microgels to stabilize emulsions. However, whatever the interfacial tension, metastable emulsions were obtained. Conversely, we found that the most important parameter governing emulsion mechanical stability is microgel deformability. Such finding is in line with previous data regarding pNIPAM, but we demonstrate for the first time, that these principles are applicable to a broad range of microgel chemistries. In contrast, we raise a new question regarding emulsion stability upon environmental changes. Until now, it was generally admitted that microgel deswelling was a simple way to trigger demulsification $[2,6,10,14,80]$. The present work demonstrates that this link is not systematic. This observation sheds light on the poor understanding of the coalescence process and the necessity for further investigations. In particular, it would be fruitful to study in depth the role of temperature on the interfacial behavior of these microgels.

On a practical point of view, the outstanding stability of pOEMA emulsions upon temperature rise could be used advantageously in applications where one needs to decouple the microgels phase transition from the emulsion stability. For instance, it is known that many properties of microgels are enhanced at the phase transition, such as the luminescent [85], electrochemical [86] or catalytic [87] properties. It will be advantageous to exploit them without destroying the emulsion. 


\section{Acknowledgements}

The authors thank the "Ministère de l'Enseignement supérieur, de la Recherche et de I'Innovation" for funding the fellowship of M.-C. Tatry. The authors also thank the PLACAMAT imaging facility for cryoSEM images.

\section{References}

[1] M. Karg, A. Pich, T. Hellweg, T. Hoare, L. A. Lyon, J. J. Crassous, D. Suzuki, R. A. Gumerov, S. Schneider, I. I. Potemkin and W. Richtering, Nanogels and Microgels: From Model Colloids to Applications, Recent Developments, and Future Trends, Langmuir 35(19) (2019) 6231-6255.

[2] M. Destribats, V. Lapeyre, M. Wolfs, E. Sellier, F. Leal-Calderon, V. Ravaine and V. Schmitt, Soft microgels as Pickering emulsion stabilisers: role of particle deformability Soft Matter 7 (2011) 7689-7698

[3] V. Schmitt and V. Ravaine, Surface compaction versus stretching in Pickering emulsions stabilised by microgels, Curr. Opin. Colloid Interface Sci. 18(6) (2013) 532-541.

[4] M. Rey, M. A. Fernandez-Rodriguez, M. Karg, L. Isa and N. Vogel, Poly-Nisopropylacrylamide Nanogels and Microgels at Fluid Interfaces, Acc. Chem. Res. 53(2) (2020) 414-424.

[5] T. Ngai, H. Auweter and S. H. Behrens, Environmental responsiveness of microgel particles and particle-stabilized emulsions, Macromolecules 39(23) (2006) 8171. 
[6] T. Ngai, S. H. Behrens and H. Auweter, Novel emulsions stabilized by $\mathrm{pH}$ and temperature sensitive microgels, Chem. Commun. (3) (2005) 331.

[7] S. Tsuji and H. Kawaguchi, Thermosensitive pickering emulsion stabilized by poly(Nisopropylacrylamide)-carrying particles, Langmuir 24(7) (2008) 3300.

[8] B. Brugger and W. Richtering, Emulsions stabilized by stimuli-sensitive poly(Nisopropylacrylamide)-co- methacrylic acid polymers: Microgels versus low molecular weight polymers, Langmuir 24(15) (2008) 7769.

[9] S. Wiese, Y. Tsvetkova, N. J. E. Daleiden, A. C. Spieß and W. Richtering, Microgel stabilized emulsions: Breaking on demand, Colloids Surf., A 495 (2016) 193-199.

[10] M.-h. Kwok, J. Ambreen and T. Ngai, Correlating the effect of co-monomer content with responsiveness and interfacial activity of soft particles with stability of corresponding smart emulsions, J. Colloid Interface Sci. 546 (2019) 293-302.

[11] L. Braun, M. Kühnhammer and R. von Klitzing, Stabvility of aqueous foam films and foams containing polymers: Discrepancies between different length scales, Curr. Opin. Colloid Interface Sci. 50 (2020) 101379.

[12] Y. Horiguchi, H. Kawakita, K. Ohto and S. Morisada, Temperature-responsive Pickering foams stabilized by poly(N-isopropylacrylamide) nanogels, Advanced Powder Technology 29(2) (2018) 266-272.

[13] S. Schmidt, T. Liu, S. Rütten, K.-H. Phan, M. Moeller and W. Richtering, Influence of Microgel Architecture and Oil Polarity on Stabilization of Emulsions by Stimuli-Sensitive Core-Shell Poly(N-isopropyl Acrylamide-co-Methacrylic Acid) / P(NiPAM-co-MAA) Microgels: Mickering vs. Pickering Behavior?, Langmuir 27(16) (2011) 9801.

[14] W. Richtering, Responsive emulsions stabilized by stimuli-sensitive microgels: Emulsions with special non-pickering properties, Langmuir 28(50) (2012) 17218-17229. 
[15] H. G. Schild, Poly(N-isopropylacrylamide): Experiment, theory and application, Progress in Polymer Science (Oxford) 17(2) (1992) 163.

[16] J. Zhang and R. Pelton, Poly(N-isopropylacrylamide) microgels at the air-water interface, Langmuir 15(23) (1999) 8032-8036.

[17] B. Brugger, S. Rütten, K. H. Phan, M. Möller and W. Richtering, The colloidal suprastructure of smart microgels at oil- Water interfaces, Angew. Chem., Int. Ed. 48(22) (2009) 3978 .

[18] H. Minato, M. Murai, T. Watanabe, S. Matsui, M. Takizawa, T. Kureha and D. Suzuki, The deformation of hydrogel microspheres at the air/water interface, Chem. Commun. 54(8) (2018) 932-935.

[19] K. Geisel, L. Isa and W. Richtering, Unraveling the 3D localization and deformation of responsive microgels at oil/water interfaces: A step forward in understanding soft emulsion stabilizers, Langmuir 28(45) (2012) 15770-15776.

[20] K. Zielińska, H. Sun, R. A. Campbell, A. Zarbakhsh and M. Resmini, Smart nanogels at the air/water interface: structural studies by neutron reflectivity, Nanoscale 8(9) (2016) 49514960.

[21] A. M. Rumyantsev, R. A. Gumerov and I. I. Potemkin, A polymer microgel at a liquidliquid interface: theory vs. computer simulations, Soft Matter 12(32) (2016) 6799-6811.

[22] L. Keal, V. Lapeyre, V. Ravaine, V. Schmitt and C. Monteux, Drainage dynamics of thin liquid foam films containing soft PNiPAM microgels: influence of the cross-linking density and concentration, Soft Matter 13(1) (2017) 170-180.

[23] C. Picard, P. Garrigue, M.-C. Tatry, V. Lapeyre, S. Ravaine, V. Schmitt and V. Ravaine, Organization of Microgels at the Air-Water Interface under Compression: Role of Electrostatics and Cross-Linking Density, Langmuir 33(32) (2017) 7968-7981. 
[24] R. W. Style, L. Isa and E. R. Dufresne, Adsorption of soft particles at fluid interfaces, Soft Matter 11(37) (2015) 7412-7419.

[25] B. Brugger, J. Vermant and W. Richtering, Interfacial layers of stimuli-responsive poly(N-isopropylacrylamide-co- methacrylicacid) (PNIPAM-co-MAA) microgels characterized by interfacial rheology and compression isotherms, Phys. Chem. Chem. Phys. 12(43) (2010) 14573-14578.

[26] K. Geisel, L. Isa and W. Richtering, The compressibility of ph-sensitive microgels at the oil-water interface: Higher charge leads to less repulsion, Angew. Chem., Int. Ed. 53(19) (2014) 4905-4909.

[27] M. Rey, M. Á. Fernández-Rodríguez, M. Steinacher, L. Scheidegger, K. Geisel, W. Richtering, T. M. Squires and L. Isa, Isostructural solid-solid phase transition in monolayers of soft core-shell particles at fluid interfaces: Structure and mechanics, Soft Matter 12(15) (2016) $3545-3557$.

[28] L. Scheidegger, M. A. Fernandez-Rodriguez, K. Geisel, M. Zanini, R. Elnathan, W. Richtering and L. Isa, Compression and deposition of microgel monolayers from fluid interfaces: particle size effects on interface microstructure and nanolithography, Phys. Chem. Chem. Phys. 19(13) (2017) 8671-8680.

[29] M. Rey, X. Hou, J. S. J. Tang and N. Vogel, Interfacial arrangement and phase transitions of PNiPAm microgels with different crosslinking densities, Soft Matter 13(46) (2017) 87178727.

[30] N. Vogel, C. Fernández-López, J. Pérez-Juste, L. M. Liz-Marzán, K. Landfester and C. K. Weiss, Ordered arrays of gold nanostructures from interfacially assembled Au@PNIPAM hybrid nanoparticles, Langmuir 28(24) (2012) 8985-8993.

[31] T. Honold, K. Volk, A. Rauh, J. P. S. Fitzgerald and M. Karg, Tunable plasmonic surfaces via colloid assembly, J. Mater. Chem. C 3(43) (2015) 11449-11457. 
[32] A. Rauh, M. Rey, L. Barbera, M. Zanini, M. Karg and L. Isa, Compression of hard coresoft shell nanoparticles at liquid-liquid interfaces: influence of the shell thickness, Soft Matter (2016).

[33] F. Pinaud, K. Geisel, P. Masse, B. Catargi, L. Isa, W. Richtering, V. Ravaine and V. Schmitt, Adsorption of microgels at an oil-water interface: correlation between packing and 2D elasticity, Soft Matter 10 (2014) 6963-6974.

[34] M. C. Tatry, E. Laurichesse, A. Perro, V. Ravaine and V. Schmitt, Kinetics of spontaneous microgels adsorption and stabilization of emulsions produced using microfluidics, J. Colloid Interface Sci. 548 (2019) 1-11.

[35] M.-C. Tatry, Y. Qiu, V. Lapeyre, P. Garrigue, V. Schmitt and V. Ravaine, Sugarresponsive Pickering emulsions mediated by switching hydrophobicity in microgels, J. Colloid Interface Sci. 561 (2020) 481-493.

[36] P. Massé, E. Sellier, V. Schmitt and V. Ravaine, Impact of electrostatics on the adsorption of microgels at the interface of pickering emulsions, Langmuir 30(49) (2014) 14745-14756.

[37] J. Maldonado-Valderrama, T. Del Castillo-Santaella, I. Adroher-Benítez, A. MonchoJordá and A. Martín-Molina, Thermoresponsive microgels at the air-water interface: the impact of the swelling state on interfacial conformation, Soft Matter 13(1) (2017) 230-238.

[38] B. S. Murray, Microgels at fluid-fluid interfaces for food and drinks, Adv. Colloid Interface Sci. 271 (2019) 101990.

[39] E. Dickinson, Biopolymer-based particles as stabilizing agents for emulsions and foams, Food Hydrocolloids 68 (2017) 219-231.

[40] J. V. M. Benetti, J. T. do Prado Silva and V. R. Nicoletti, SPI microgels applied to Pickering stabilization of $\mathrm{O} / \mathrm{W}$ emulsions by ultrasound and high-pressure homogenization: rheology and spray drying, Food Res. Int. 122 (2019) 383-391. 
[41] B. Jiao, A. Shi, Q. Wang and B. P. Binks, High-Internal-Phase Pickering Emulsions Stabilized Solely by Peanut-Protein-Isolate Microgel Particles with Multiple Potential Applications, Angew. Chem., Int. Ed. 57(30) (2018) 9274-9278.

[42] M. Destribats, M. Rouvet, C. Gehin-Delval, C. Schmitt and B. P. Binks, Emulsions stabilised by whey protein microgel particles: towards food-grade Pickering emulsions, Soft Matter 10(36) (2014) 6941-6954.

[43] R. W. Murphy, B. E. Farkas and O. G. Jones, Dynamic and viscoelastic interfacial behavior of $\beta$-lactoglobulin microgels of varying sizes at fluid interfaces, J. Colloid Interface Sci. 466 (2016) 12-19.

[44] X. Li, Y. Yang, B. S. Murray and A. Sarkar, Combination of egg white protein and microgels to stabilize foams: Impact of processing treatments, J. Food Eng. 275 (2020) 109860. [45] G. I. Saavedra Isusi, B. Bindereif, H. P. Karbstein and U. S. van der Schaaf, Polymer or microgel particle: Differences in emulsifying properties of pectin as microgel or as individual polymer chains, Colloids Surf., A 598 (2020) 124793.

[46] J.-F. Lutz, Ö. Akdemir and A. Hoth, Point by Point Comparison of Two Thermosensitive Polymers Exhibiting a Similar LCST: Is the Age of Poly(NIPAM) Over?, J. Am. Chem. Soc. 128(40) (2006) 13046-13047.

[47] J.-F. Lutz and A. Hoth, Preparation of Ideal PEG Analogues with a Tunable Thermosensitivity by Controlled Radical Copolymerization of 2-(2-Methoxyethoxy)ethyl Methacrylate and Oligo(ethylene glycol) Methacrylate, Macromolecules 39(2) (2006) 893-896. [48] J. F. Lutz, Polymerization of oligo(ethylene glycol) (meth)acrylates: Toward new generations of smart biocompatible materials, J. Polym. Sci., Part A: Polym. Chem. 46(11) (2008) 3459-3470.

[49] G. Vancoillie, D. Frank and R. Hoogenboom, Thermoresponsive poly(oligo ethylene glycol acrylates), Prog. Polym. Sci. 39(6) (2014) 1074-1095. 
[50] N. Badi, Non-linear PEG-based thermoresponsive polymer systems, Prog. Polym. Sci. 66 (2017) 54-79.

[51] T. Cai, M. Marquez and Z. Hu, Monodisperse Thermoresponsive Microgels of Poly(ethylene glycol) Analogue-Based Biopolymers, Langmuir 23(17) (2007) 8663-8666.

[52] N. Welsch and L. A. Lyon, Oligo(ethylene glycol)-sidechain microgels prepared in absence of cross-linking agent: Polymerization, characterization and variation of particle deformability, PLoS One 12(7) (2017) e0181369.

[53] L. Hou and P. Wu, Microgels with Linear Thermosensitivity in a Wide Temperature Range, Macromolecules 49(16) (2016) 6095-6100.

[54] K. Gawlitza, A. Radulescu, R. von Klitzing and S. Wellert, On the structure of biocompatible, thermoresponsive poly(ethylene glycol) microgels, Polymer 55(26) (2014) $6717-6724$.

[55] S. Wellert, A. Radulescu, A. Carl, R. v. Klitzing and K. Gawlitza, Evolution of Size and Structure during the Polymerization Process: A SANS Study on EG-Based Microgels, Macromolecules 48(14) (2015) 4901-4909.

[56] M. Boularas, E. Gombart, J. F. Tranchant, L. Billon and M. Save, Design of smart oligo(ethylene glycol)-based biocompatible hybrid microgels loaded with magnetic nanoparticles, Macromol. Rapid Commun. 36(1) (2015) 79-83.

[57] G. Aguirre, E. Deniau, A. Brûlet, K. Chougrani, V. Alard and L. Billon, Versatile oligo(ethylene glycol)-based biocompatible microgels for loading/release of active bio(macro)molecules, Colloids Surf, B. 175 (2019) 445-453.

[58] G. Aguirre, A. Khoukh, P. Taboada, K. Chougrani, V. Alard and L. Billon, Smart selfassembled microgel films as encapsulating carriers for UV-absorbing molecules, Polym. Chem. 9(10) (2018) 1155-1159. 
[59] M. Boularas, S. Radji, E. Gombart, J.-F. Tranchant, V. Alard and L. Billon, Functional film by trigger-free self-assembly of adhesive soft microgels at skin temperature, Materials \& Design 147 (2018) 19-27.

[60] R. H. Pelton and P. Chibante, Preparation of aqueous latices with N-isopropylacrylamide, Colloids Surf. 20(3) (1986) 247.

[61] M. Boularas, E. Deniau-Lejeune, V. Alard, J.-F. Tranchant, L. Billon and M. Save, Dual stimuli-responsive oligo(ethylene glycol)-based microgels: insight into the role of internal structure in volume phase transitions and loading of magnetic nanoparticles to design stable thermoresponsive hybrid microgels, Polym. Chem. 7(2) (2016) 350-363.

[62] S. Arditty, C. P. Whitby, B. P. Binks, V. Schmitt and F. Leal-Calderon, Some general features of limited coalescence in solid-stabilized emulsions, Eur. Phys. J. E 11(3) (2003) 273. [63] O. L. J. Virtanen, H. M. Ala-Mutka and W. Richtering, Can the Reaction Mechanism of Radical Solution Polymerization Explain the Microgel Final Particle Volume in Precipitation Polymerization of N-Isopropylacrylamide?, Macromol. Chem. Phys. 216(13) (2015) 14311440.

[64] M. Keerl, J. S. Pedersen and W. Richtering, Temperature Sensitive Copolymer Microgels with Nanophase Separated Structure, J. Am. Chem. Soc. 131(8) (2009) 3093-3097.

[65] M. Rasmusson, A. Routh and B. Vincent, Flocculation of Microgel Particles with Sodium Chloride and Sodium Polystyrene Sulfonate as a Function of Temperature, Langmuir 20(9) (2004) 3536-3542.

[66] T. López-León, J. L. Ortega-Vinuesa, D. Bastos-González and A. Elaïssari, Cationic and Anionic Poly(N-isopropylacrylamide) Based Submicron Gel Particles: Electrokinetic Properties and Colloidal Stability, J. Phys. Chem. B 110(10) (2006) 4629-4636.

[67] B. R. Saunders and B. Vincent, Microgel particles as model colloids: theory, properties and applications, Adv. Colloid Interface Sci. 80(1) (1999) 1. 
[68] D. Gan and L. A. Lyon, Synthesis and protein adsorption resistance of PEG-modified poly(N-isopropylacrylamide) core/shell microgels, Macromolecules 35(26) (2002) 9634.

[69] J. Es Sayed, C. Lorthioir, P. Perrin and N. Sanson, PEGylated NiPAM microgels: synthesis, characterization and colloidal stability, Soft Matter 15(5) (2019) 963-972.

[70] S. Wellert, D. Kesal, S. Schön, R. von Klitzing and K. Gawlitza, Ethylene Glycol-Based Microgels at Solid Surfaces: Swelling Behavior and Control of Particle Number Density, Langmuir 31(7) (2015) 2202-2210.

[71] M. Destribats, V. Lapeyre, E. Sellier, F. Leal-Calderon, V. Ravaine and V. Schmitt, Origin and control of adhesion between emulsion drops stabilized by thermally sensitive soft colloidal particles, Langmuir 28(8) (2012) 3744-3755.

[72] M. Destribats, V. Lapeyre, E. Sellier, F. Leal-Calderon, V. Schmitt and V. Ravaine, Waterin-oil emulsions stabilized by water-dispersible poly( $\mathrm{N}$ - isopropylacrylamide) microgels: Understanding anti-Finkle behavior, Langmuir 27(23) (2011) 14096-14107.

[73] C. Monteux, C. Marliére, P. Paris, N. Pantoustier, N. Sanson and P. Perrin, Poly(Nisopropylacrylamide) microgels at the oil-water interface: Interfacial properties as a function of temperature, Langmuir 26(17) (2010) 13839-13846.

[74] J. Harrer, M. Rey, S. Ciarella, H. Löwen, L. M. C. Janssen and N. Vogel, StimuliResponsive Behavior of PNiPAm Microgels under Interfacial Confinement, Langmuir 35(32) (2019) 10512-10521.

[75] M. Destribats, M. Eyharts, V. Lapeyre, E. Sellier, I. Varga, V. Ravaine and V. Schmitt, Impact of pNIPAM Microgel Size on Its Ability To Stabilize Pickering Emulsions, Langmuir 30(7) (2014) 1768-1777.

[76] M.-h. Kwok and T. Ngai, A confocal microscopy study of micron-sized poly(Nisopropylacrylamide) microgel particles at the oil-water interface and anisotopic flattening of highly swollen microgel, J. Colloid Interface Sci. 461 (2016) 409-418. 
[77] M.-h. Kwok and T. Ngai, Comparing the Relative Interfacial Affinity of Soft Colloids With Different Crosslinking Densities in Pickering Emulsions, Frontiers in Chemistry 6(148) (2018).

[78] S. Kawano, T. Kida, M. Akashi, H. Sato, M. Shizuma and D. Ono, Preparation of Pickering emulsions through interfacial adsorption by soft cyclodextrin nanogels, Beilstein J. Org. Chem. $11(2015)$ 2355-2364.

[79] Y. Wu, S. Wiese, A. Balaceanu, W. Richtering and A. Pich, Behavior of TemperatureResponsive Copolymer Microgels at the Oil/Water Interface, Langmuir 30(26) (2014) 76607669.

[80] A. J. Morse, E. C. Giakoumatos, S.-Y. Tan, G. B. Webber, S. P. Armes, S. Ata and E. J. Wanless, Giant pH-responsive microgel colloidosomes: preparation, interaction dynamics and stability, Soft Matter 12(5) (2016) 1477-1486.

[81] W. Wang, A. H. Milani, Z. Cui, M. Zhu and B. R. Saunders, Pickering Emulsions Stabilized by $\mathrm{pH}$-Responsive Microgels and Their Scalable Transformation to Robust Submicrometer Colloidoisomes with Selective Permeability, Langmuir 33(33) (2017) 81928200.

[82] S. Zhang, M. Holmes, R. Ettelaie and A. Sarkar, Pea protein microgel particles as Pickering stabilisers of oil-in-water emulsions: Responsiveness to $\mathrm{pH}$ and ionic strength, Food Hydrocolloids 102 (2020) 105583.

[83] Z. Wang, N. Zhang, C. Chen, R. He and X. Ju, Rapeseed Protein Nanogels As Novel Pickering Stabilizers for Oil-in-Water Emulsions, J. Agric. Food Chem. 68(11) (2020) 36073614.

[84] K. Matsumiya and B. S. Murray, Soybean protein isolate gel particles as foaming and emulsifying agents, Food Hydrocolloids 60 (2016) 206-215. 
[85] L. Beauté, N. McClenaghan and S. Lecommandoux, Photo-triggered polymer nanomedicines: From molecular mechanisms to therapeutic applications, Adv Drug Delivery Rev 138 (2019) 148-166.

[86] R. Bois, S. Scarabino, V. Ravaine and N. Sojic, Two-Dimensional Electrochemiluminescence: Light Emission Confined at the Oil-Water Interface in Emulsions Stabilized by Luminophore-Grafted Microgels, Langmuir 33(29) (2017) 7231-7238.

[87] H. Jia, J. Cao and Y. Lu, Design and fabrication of functional hybrid materials for catalytic applications, Curr. Opin. Green Sustain. Chem. 4 (2017) 16-22. 